\title{
Methodology for the Determination of Trace and Minor Elements in Minerals and Fused Rock Glasses with Laser Ablation Associated with Quadrupole Inductively Coupled Plasma Mass Spectrometry (LA-Q-ICPMS)
}

\author{
Sandra Andrade*, Horstpeter Herberto Ulbrich, Celso de Barros Gomes, \\ Lucelene Martins \\ Department of Mineralogy and Geotectonics, Institute of Geosciences, University of São Paulo, São Paulo, \\ Brazil \\ Email:
}

Received 24 May 2014; revised 13 July 2014; accepted 28 July 2014

Copyright (C) 2014 by authors and Scientific Research Publishing Inc.

This work is licensed under the Creative Commons Attribution International License (CC BY).

http://creativecommons.org/licenses/by/4.0/

(c) (i) Open Access

\begin{abstract}
The laser ablation technique, coupled with the use of quadrupole ICPMS equipment, proved a powerful tool for determination of trace elements in minerals. At the University of São Paulo, the technique was implemented for the study of minerals such as olivines, pyroxenes and biotites. The main problem to be tackled is the availability of proper multi-element reference materials usually prepared synthetically as glasses with various compositions by NIST and fused rock glasses by the Max Planck Institute (MPI) and USGS (basalts, andesite, quartz diorite, komatiites). The best tested ones are the NIST glasses, with good homogeneity and reliable compositional data for over 40 elements. Results are here presented that test additional RM's. NIST 612 and 610 were used for calibration purposes. The best results were obtained for rock glasses USGS basalts BHVO-2G, BIR$1 G$ and BCR-2G (better homogeneity and recommended values). Our contribution tests especially the MPI komatiites glasses GOR-128 and GOR-132G, basalts KL-2G and ML-3BG, andesite StHs-6/ 80G and quartz diorite T-1G, discussing homogeneity issues and providing new data. There is a need for additional preparation of reliable reference materials.
\end{abstract}

\section{Keywords}

Laser Ablation, Mass Spectrometry, Geological Reference Materials, Fused Rock Glasses

\footnotetext{
*Corresponding author.
}

How to cite this paper: Andrade, S., Ulbrich, H.H., de Barros Gomes, C. and Martins, L. (2014) Methodology for the Determination of Trace and Minor Elements in Minerals and Fused Rock Glasses with Laser Ablation Associated with Quadrupole Inductively Coupled Plasma Mass Spectrometry (LA-Q-ICPMS). American Journal of Analytical Chemistry, 5, 701-721. 


\section{Introduction}

Inductively coupled plasma mass spectrometry, hyphenated with laser ablation (LA-ICPMS), represents an important tool for the direct chemical analyses in solid phases. The main advantage is the possibility of determining the composition even in substances with a complex matrix, as usually present in geologic and environmental materials, semi-conductors, and others. The technique has the advantage of high sensitivity, a wide range of linear intensity responses, and requiring little surface preparation, without presenting major problems with contamination issues. The obtained results can be expressed as either elemental or isotopic proportions and the spatial resolution is of the order of 10 to $300 \mu \mathrm{m}$ and depth resolution is about $60 \mathrm{~nm}$ per pulse [1].

Mass spectrometers can isolate ions with a positive charge effectively by their mass/charge relations. Three types of equipments are available, each one with its own advantages and shortcomings, the quadrupole setting, the time-of-flight performance and the high-resolution sector arrangement.

The quadrupole mass spectrometer setting is the most cost-effective, and hence has become the preferred instrument in many laboratories. The equipment generates voltage intervals and radiofrequencies that can stabilize a definite ion within the quadrupole rods and guide it towards the detector window. Given a predefined setting, ions with a different mass-to-charge ratio will become unstable and be eliminated within the vacuum system. All the desired spectra of ionic masses can be quickly scanned within the quadrupole. Although this analyzer works in a sequential fashion, it can separate efficiently more than 5000 atomic mass units (amu) per second [2].

Therefore, Q-ICPMS equipments associated with laser ablation can perform very fast multi-element determinations of over 40 elements, with sensitivities of the order of $\mathrm{ng} \cdot \mathrm{g}^{-1}$ in any mineral phase, or of materials hidden within them, such as fluid inclusions, in a time-span that does not exceed 1 or 2 minutes of operation.

These techniques opened up new fields of research in igneous petrology, mineralogy and geochronology, determining the distribution of trace elements in several phases (both solid as well as liquid), and enabling also the calculation of the corresponding partition coefficients between mineral pairs, or mineral phases and melts [3] [4].

The main drawback of the quadrupole setting associated with LA is the possibility of isotopic fractionation during analysis, a subject widely discussed in the literature [5] [6], as well as the lack of adequate Certified Reference Materials (CRM) for most of the analyzed minerals, a deficiency that may be corrected with the preparation of new homogeneous synthetic materials [7]. Many such multi-elementary synthetic or natural materials are already available, such as the well-certified NIST 610 and 612 glasses and the synthetic materials prepared by melting of rock powders [8] [9]. The NIST RM's are synthetic glasses with around 40 elements, present in amounts close to $30 \mathrm{mg} \cdot \mathrm{kg}^{-1}$ in the 612 sample, and up to $500 \mathrm{mg} \cdot \mathrm{kg}^{-1}$ in the 610 material. They were used in this contribution as calibration materials. The USGS substances, widely considered the best RM's, are the basaltic glasses BHVO-2G, BIR-1G and BCR-2G, already subjected to many determinations. The Max Planck Institute prepared several additional glassy RM's, such as the komatiites GOR-128 and GOR-132G, the basalts KL-2G and ML-3BG, the andesite StHs-6/80G and the quartz diorite T-1G, still to be tested, as far as certified values and homogeneity is concerned.

The implementation of this methodology at our laboratory started with the handling and analytical determinations using NIST materials for calibration, followed by analytical determinations of the USGS synthetic basaltic glasses (BHVO-2G, BIR-1G and BCR-2G) [8], and, additionally, of the substances offered by the Max Planck Institute [10]. The results obtained for these reference materials during the last 4 years of analytical determinations in our laboratory (where these materials were also analyzed as unknown substances) are here presented and discussed.

\section{Analytical Methodology}

The indications given in [11] were used as a basis for the implementation of the analytical methodology for trace element determinations in minerals in thin section and in reference materials with the LA-Q-ICPMS methodology.

Mean analytical time spent for analyzing up to 44 elements was $120 \mathrm{~s} \mathrm{(60} \mathrm{s} \mathrm{for} \mathrm{reading} \mathrm{a} \mathrm{blank,} 60 \mathrm{~s}$ for preparing the instrument and reading results from the analyzed sample). Intensities in cps are calculated as concentrations in real time by the Glitter ${ }^{\circledR}$ software (developed by the GEMOC program, Geochemical Evolution and Metallogeny of Continents, Macquarie University, Australia) [12].

Our results were obtained from the determination of the before-mentioned RM's (USGS basalt glasses 
BHVO-2G, BIR-1G and BCR-2G; Max Planck’s komatiites GOR-128 and GOR-132G, basalts KL02G and ML03BG, quartz diorite T-1G, andesite StHs-6/80G). The obtained results were processed with the Glitter ${ }^{\circledR}$ software [12] for drift correction with the use of an internal standard, together with a check on background, spectral signs and analytical quality, and finished with the calculation of results in weight units. Instrumental parameters are listed in Table 1, and data on the analytical performance are quoted in Table 2 and Table 3.

The analyzed elements and their masses (cited in Table 3) were determined with a dwell time of 8.3ms and integration time of $1660 \mathrm{~ms}$; total time of analysis was $120 \mathrm{~s}$, the first $60 \mathrm{~s}$ measuring the background gas fluxes (Helium, He and Argon, Ar) reaching the plasma after passing through the sample cell, followed by 60 s devoted to data acquisition of the materials removed from the laser spot.

The sequence for data acquisition is as follows:

Nist-612 (a), Nist-612 (b), sample (1), $\cdots$, sample (6), Nist-612 (c), Nist-612 (d), sample (7), $\cdots$, sample (14), Nist-612 (e), Nist-612 (f).

Cleaning was performed after each sample analysis for about 2 minutes with He fluxes, a procedure that stabilizes rapidly the background values, more so than an alternative method, such as obtained with the use of Argon gas. Readings from Nist-612 (a, b, c, d, e, f) were used as an internal standard and for drift correction during the entire 1-hour analytical work. The entire procedure lasts about 3 hours, counting also laser and plasma stabilization times at the beginning of the determinations.

Isotopic interferences occurring during analysis, such as the ones due to ${ }^{28} \mathrm{Si}^{17} \mathrm{O}^{+},{ }^{27} \mathrm{Al}^{18} \mathrm{O}^{+},{ }^{90} \mathrm{Zr}^{2+}$ on ${ }^{45} \mathrm{Sc}^{+}$; ${ }^{43} \mathrm{Ca}^{16} \mathrm{O}^{+}$on ${ }^{59} \mathrm{Co}^{+} ;{ }^{44} \mathrm{Ca}^{16} \mathrm{O}^{+}$on ${ }^{60} \mathrm{Ni} ;{ }^{135} \mathrm{Ba}^{16} \mathrm{O}^{+}$on ${ }^{151} \mathrm{Eu}$, and several others, can however be ignored, since they are usually very limited in dry plasmas, with hydroxyl and oxygen absent. The daily rate of generation of oxide was controlled holding $\mathrm{ThO}^{+}$formation lower than $1 \%$. The formation of double-charged species, such as $\mathrm{Ba}^{2+}$, was kept to a maximum of $3 \%$, controlling the gas fluxes.

Table 1. Instrumental parameters used in analytical routine for determination of trace elements by LA-Q-ICPMS.

\begin{tabular}{|c|c|c|c|}
\hline ICP-MS & Elan-6100DRC & Laser Ablation & Laser New Wave UP-213 \\
\hline RF power & $1300 \mathrm{~W}$ & Type & Nd:YAG \\
\hline Auxiliary gas flux (Ar) & $1.0 \mathrm{~L} / \mathrm{min}$ & Wavelength & $213 \mathrm{~nm}$ \\
\hline Plasma gas flux (Ar) & $16 \mathrm{~L} / \mathrm{min}$ & Pulse duration & $5 \mathrm{~ns}$ \\
\hline Sample cell & Super cell & Laser repeat rate & $10 \mathrm{~Hz}$ \\
\hline Carrier gas flux & $\mathrm{Ar}=0.58 \mathrm{~L} / \mathrm{min}, \mathrm{He}=0.60 \mathrm{~L} / \mathrm{min}$ & & \\
\hline Distance to sample & $4 \mathrm{~mm}$ & & \\
\hline Pt Sampler dimension & $1.1 \mathrm{~mm}$ & & \\
\hline Pt Skimmer dimension & $0.9 \mathrm{~mm}$ & & \\
\hline Ionic lens voltage & 5.5 to $8.5 \mathrm{~V}$ & & \\
\hline Energy density & \multicolumn{3}{|c|}{$65 \%$ laser power $\sim 8.60 \mathrm{~J} / \mathrm{cm}^{2}$ and $0.2 \mathrm{~mJ}$ (spot $65 \mu \mathrm{m}$ ) for pyroxenes, amphiboles } \\
\hline Energy density & \multicolumn{3}{|c|}{$\begin{array}{l}70 \% \text { laser power } \sim 7-10 \mathrm{~J} / \mathrm{cm}^{2} \text { and } 0.2-0.3 \mathrm{~mJ} \text { (raster with spot of } 65 \mu \mathrm{m} \text { ) } \\
\text { Ablation speed } 5 \mu \mathrm{m} / \mathrm{s} \text {, line of } 300 \mu \mathrm{m} \text { for quartz, alkali feldspars }\end{array}$} \\
\hline
\end{tabular}

Table 2. Time parameters and signal processing used for determinations of trace elements.

\begin{tabular}{cccc}
\hline ICP-Q-MS & Parameters & ICP-Q-MS & Parameters \\
\hline Sweeps/reading & 1 & Number of replicates & 1 \\
Readings/replicates & 270 & Settling time & MCA channel \\
Scan mode & Peak hopping & Units & Cps \\
Detector & Analogic pulse & Mode & Standard (without DRC) \\
Auto-lens & ON &
\end{tabular}


Table 3. Analyzed elements and their isotope masses (time parameters of signal integration, see text).

\begin{tabular}{|c|c|c|c|}
\hline Element & Mass (u.m.a) & Element & Mass (u.m.a) \\
\hline $\mathrm{Li}$ & 7 & Sn & 118 \\
\hline $\mathrm{Be}$ & 9 & $\mathrm{Sb}$ & 121 \\
\hline $\mathrm{Mg}$ & 25 & Cs & 133 \\
\hline $\mathrm{Si}$ & 29 & $\mathrm{Ba}$ & 137 \\
\hline $\mathrm{P}$ & 31 & $\mathrm{La}$ & 139 \\
\hline $\mathrm{Ca}$ & 42 & $\mathrm{Ce}$ & 140 \\
\hline Sc & 45 & $\operatorname{Pr}$ & 141 \\
\hline $\mathrm{Ti}$ & 49 & $\mathrm{Nd}$ & 143 \\
\hline $\mathrm{V}$ & 51 & $\mathrm{Sm}$ & 147 \\
\hline $\mathrm{Cr}$ & 52 & $\mathrm{Eu}$ & 151 \\
\hline Mn & 55 & Gd & 155 \\
\hline Co & 59 & $\mathrm{~Tb}$ & 159 \\
\hline $\mathrm{Ni}$ & 60 & Dy & 163 \\
\hline $\mathrm{Cu}$ & 65 & Но & 165 \\
\hline $\mathrm{Zn}$ & 66 & Er & 166 \\
\hline $\mathrm{Ga}$ & 71 & $\mathrm{Tm}$ & 169 \\
\hline $\mathrm{Rb}$ & 85 & $\mathrm{Yb}$ & 173 \\
\hline $\mathrm{Sr}$ & 88 & $\mathrm{Lu}$ & 175 \\
\hline $\mathrm{Y}$ & 89 & $\mathrm{Hf}$ & 179 \\
\hline $\mathrm{Zr}$ & 90 & Ta & 181 \\
\hline $\mathrm{Nb}$ & 93 & $\mathrm{~Pb}$ & 208 \\
\hline \multirow[t]{2}{*}{ Mo } & 95 & Th & 232 \\
\hline & & $\mathrm{U}$ & 238 \\
\hline
\end{tabular}

Determination of elemental concentrations can be achieved by means of any of the three cited methods:

1) Entirely by external calibration with a solid reference material;

2) External calibration with a solid reference material coupled with internal standardization;

3) Calibration using solutions.

In our laboratory, the second type of calibration was implemented. The external reference material was used simultaneously with an internal standard. This procedure represents a more robust calibration method, since a correction can be applied on the ablation yield of the sample (the mass of removed material) and the external reference material. The methodology is especially adapted for use with the Glitter ${ }^{\circledR}$ software [13]. In addition, matrix effects and signal drift can also be corrected. In practical terms, the internal standard is represented by a major constituent found in known concentrations both in the sample and the reference material. Calcium, in particular, has proven to be an excellent internal standard for many minerals and the NIST glasses. The three minor isotopes of the element $(42,43,44)$ can be used better for calibration purposes, since their ablation behavior is similar to the one shown by petrogenetically important minor elements (e.g., REE). For correction purposes, it is assumed that the signal drift occurs linearly with time and at a similar rate for all isotopic masses. When Ca is present in concentrations below 1\%, other elements can be used as internal standard, such as Mg our even Si.

The concentration of elements, even though calculated directly with the Glitter ${ }^{\circledR}$ software [12], can also be obtained by defining the intensity integration points for background and the sample analyzed by laser, using the 
Equation (1):

$$
C_{\mathrm{AN} \text { sample }}=\frac{I_{A N_{\text {sample }}}}{S}
$$

where:

$C_{A N_{\text {sample }}}$ is the concentration of the analyzed element in the sample,

$I_{A N_{\text {sample }}}$ is the intensity in cps of the element in the sample and $S$ is the normalized sensitivity, given by Equation (2):

$$
S=\frac{I_{A N_{\text {reference }}}}{C_{A N_{\text {reference }}}}\left(\frac{I_{S I_{\text {sample }}}}{I_{S I_{\text {reference }}}} \times \frac{C_{S I_{\text {reference }}}}{C_{S I_{\text {sample }}}}\right)
$$

where:

$I_{A N_{\text {reference }}}$ is the intensity in cps of the element in the reference material,

$C_{\mathrm{AN}_{\text {reference }}}$ is the concentration of the element in the reference material,

$I_{S I_{\text {sample }}}$ and $I_{S I_{\text {reference }}}$ are the intensities in cps of the internal standard (Ca) in the sample and reference material, respectively,

$C_{S I_{\text {sample }}}$ and $C_{S I_{\text {reference }}}$ are the concentrations of the internal standard (Ca) in the sample and reference material, respectively.

Calculation of this detection limit was defined [13] by Equation (3), below:

$$
\mathrm{DL}=\frac{3 \sigma_{B C G}}{S \times Y} \times \sqrt{\frac{1}{N_{B C G}}+\frac{1}{N_{P K}}} .
$$

where $\sigma_{B C G}$ is the standard deviation of replicate analysis of the pre-ablation background identification; $N_{B C G}$ and $N_{P K}$ are the number of replicate determinations used for background and peak signal integration, respectively; $S$ is the normalized sensitivity in cps per unit of concentration for the reference material (see Equation (1)); $Y$ the ablation yield relative to the reference material, determined from the counting intensity measurement and the known concentration of the internal standard.

Detection limits of about tens of $\mathrm{ng} \cdot \mathrm{g}^{-1}$ are expected for present-day instruments, for a moderate spatial peak resolution between 30 to $40 \mu \mathrm{m}$. Higher spatial resolutions (of the order of $10 \mu \mathrm{m}$ ) will present detection limits at the $\mathrm{mg} \cdot \mathrm{kg}^{-1}$ range, lower resolutions $(>100 \mu \mathrm{m})$ at a sub-ng $\cdot \mathrm{g}^{-1}$ range.

Detection limits are calculated for each element in each sample directly by the Glitter ${ }^{\circledR}$ software (cf. Equation (3) [13]). In Table 4 are presented average values and standard deviations of detection limits obtained for elements in several analytical runs.

\section{Obtained Results}

Data obtained during the last 4 years in our laboratory for USGS basaltic glasses BCR-2G, BIR-1G and BHVO2G are listed in Tables 5-7. These RM's were used as quality control standards with 30 to 60 obtained values for each analyzed element. Reference values for LA-ICPMS are quoted in [11] and in [10]. In the GEOREM site [14] preferred values are also listed with a mention of the used analytical technique.

Data obtained for the Max Planck Institute basaltic glasses KL-2G and ML-3BG, andesite StHs-6/80G, quartz diorite T-1G and komatiites GOR-128 and GOR-132G are quoted in Tables 8-13 (six to twelve determinations for each material).

Data obtained for materials BHVO-2G, BCR-2G and BIR-1G were calibrated against the synthetic glasses Nist-610 or 612, while the RM from the Max Planck Institute were calibrated exclusively against Nist-612.

\section{Discussion of Results}

The obtained values for the analyzed elements (Tables 5-7) in the basaltic glasses BHVO-2G, BCR-2G and BIR-1G are represented in graphical form in Figure 1, with variations found in analytical results obtained in 
Table 4. Mean detection limits ( $\mathrm{mg} \cdot \mathrm{kg}^{-1}$ ) and standard deviations (sd) obtained in several analytical runs.

\begin{tabular}{|c|c|c|c|c|c|c|c|c|}
\hline Isotope & DL & sd & Isotope & $\mathrm{DL}$ & sd & Isotope & $\mathrm{DL}$ & sd \\
\hline${ }^{7} \mathrm{Li}$ & 0.53 & 0.101 & ${ }^{88} \mathrm{Sr}$ & 0.05 & 0.008 & ${ }^{159} \mathrm{~Tb}$ & 0.03 & 0.003 \\
\hline${ }^{9} \mathrm{Be}$ & 3.05 & 0.264 & ${ }^{89} \mathrm{Y}$ & 0.04 & 0.007 & ${ }^{163}$ Dy & 0.12 & 0.011 \\
\hline${ }^{25} \mathrm{Mg}$ & 1.40 & 0.267 & ${ }^{90} \mathrm{Zr}$ & 0.07 & 0.014 & ${ }^{165} \mathrm{Ho}$ & 0.03 & 0.004 \\
\hline${ }^{31} \mathrm{P}$ & 19.74 & 2.973 & ${ }^{93} \mathrm{Nb}$ & 0.05 & 0.006 & ${ }^{166} \mathrm{Er}$ & 0.09 & 0.015 \\
\hline${ }^{42} \mathrm{Ca}$ & 0.03 & 0.011 & ${ }^{95} \mathrm{Mo}$ & 0.38 & 0.053 & ${ }^{169} \mathrm{Tm}$ & 0.03 & 0.002 \\
\hline${ }^{45} \mathrm{Sc}$ & 0.60 & 0.079 & ${ }^{118} \mathrm{Sn}$ & 0.70 & 0.081 & ${ }^{173} \mathrm{Yb}$ & 0.17 & 0.034 \\
\hline${ }^{49} \mathrm{Ti}$ & 2.09 & 0.182 & ${ }^{121} \mathrm{Sb}$ & 0.13 & 0.018 & ${ }^{175} \mathrm{Lu}$ & 0.03 & 0.005 \\
\hline${ }^{51} \mathrm{~V}$ & 0,45 & 0.089 & ${ }^{133} \mathrm{Cs}$ & 0.04 & 0.005 & ${ }^{179} \mathrm{Hf}$ & 0.20 & 0.012 \\
\hline${ }^{52} \mathrm{Cr}$ & 2.43 & 0.202 & ${ }^{137} \mathrm{Ba}$ & 0.27 & 0.050 & ${ }^{181} \mathrm{Ta}$ & 0.03 & 0.004 \\
\hline${ }^{55} \mathrm{Mn}$ & 0.61 & 0.281 & ${ }^{139} \mathrm{La}$ & 0.04 & 0.005 & ${ }^{208} \mathrm{~Pb}$ & 0.09 & 0.009 \\
\hline${ }^{59} \mathrm{Co}$ & 0.11 & 0.018 & ${ }^{140} \mathrm{Ce}$ & 0.03 & 0.007 & ${ }^{232} \mathrm{Th}$ & 0.03 & 0.001 \\
\hline${ }^{60} \mathrm{Ni}$ & 0.52 & 0.085 & ${ }^{141} \mathrm{Pr}$ & 0.03 & 0.004 & ${ }^{238} \mathrm{U}$ & 0.03 & 0.004 \\
\hline${ }^{65} \mathrm{Cu}$ & 0.55 & 0.052 & ${ }^{143} \mathrm{Nd}$ & 0.23 & 0.031 & & & \\
\hline${ }^{66} \mathrm{Zn}$ & 1.43 & 0.313 & ${ }^{147} \mathrm{Sm}$ & 0.19 & 0.031 & & & \\
\hline${ }^{71} \mathrm{Ga}$ & 0.19 & 0.056 & ${ }^{151} \mathrm{Eu}$ & 0.06 & 0.009 & & & \\
\hline${ }^{85} \mathrm{Rb}$ & 0.11 & 0.020 & ${ }^{155} \mathrm{Gd}$ & 0.24 & 0.050 & & & \\
\hline
\end{tabular}

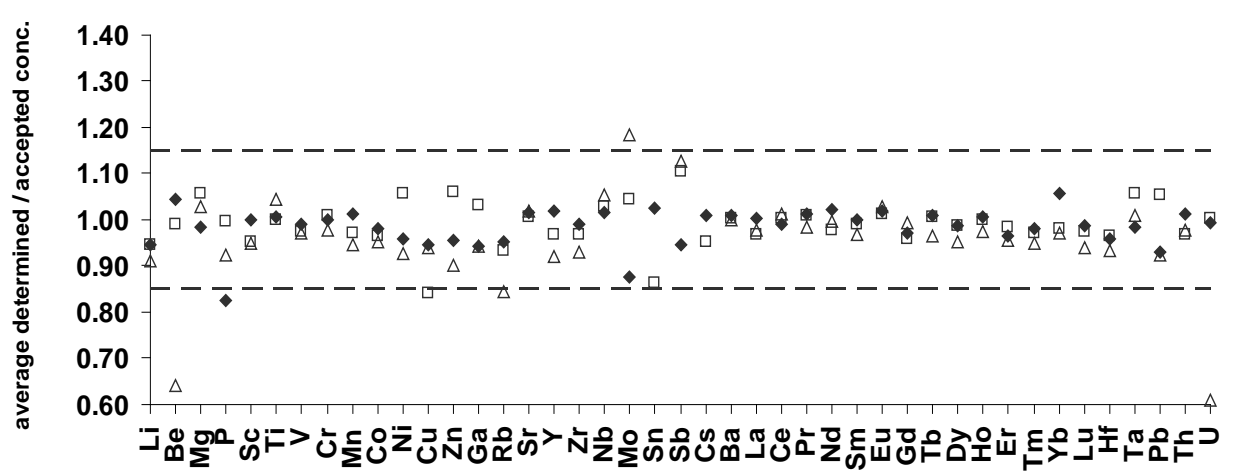

- BCR-2G $\square$ BHVO-2G $\triangle$ BIR-1G

Figure 1. Relationship between average values and recommended values [11], determined with the LA-Q-ICPMS technique for the analyzed elements in the BCR-2G, BHVO-2G and BIR-1G basaltic glasses (cf. Tables 5-7).

relation to the preferred mean, taking as mean values the ones quoted in [11], determined with the LA-Q-ICPMS technique. Variation diagrams for REE normalized with respect to values of C1 chondrite ([16] and [17]), are presented in Figure 2.

Most obtained values for the referred glasses lie within an interval of $\pm 15 \%$ of the reference values of the cited elements, as determined by the LA-Q-ICPMS technique in [11]. The data in Figure 2 also indicate a general compliance with the published contents for the REE elements in three reference materials. Beryllium, uranium and Mo for the BIR-1G and P for BCR-2G are the elements showing the highest deviation from the recommended values. 
Table 5. Obtained values in $\mathrm{mg} \cdot \mathrm{kg}^{-1}$ for BCR-2G, compared with values quoted in [11] (LA-ICPMS technique) and preferred values in [14].

\begin{tabular}{|c|c|c|c|c|c|c|c|c|}
\hline \multirow{2}{*}{ Element } & \multicolumn{3}{|c|}{ Obtained values } & \multicolumn{3}{|c|}{ Gao et al. [11] } & \multicolumn{2}{|c|}{ GEOREM [14] } \\
\hline & Mean & sd & $\mathrm{n}$ & Mean & sd & $\mathrm{n}$ & Recom $^{* *}$ & $\mathrm{sd}^{* *}$ \\
\hline $\mathrm{Li}$ & 9.37 & 0.52 & 33 & 9.9 & 0.7 & 67 & 9 & 1 \\
\hline $\mathrm{Be}$ & 2.30 & 1.10 & 25 & 2 & 0.1 & 7 & 2.3 & 0.4 \\
\hline Mg & 21184 & 1806 & 43 & 20980 & 607 & 94 & 21470 & 1492 \\
\hline $\mathrm{P}$ & 1334 & 194 & 22 & n.d & & & 1615 & 44 \\
\hline Sc & 32.0 & 3.8 & 41 & 32 & 2 & 132 & 33 & 2 \\
\hline $\mathrm{Ti}$ & 13080 & 1182 & 43 & 13005 & 1081 & 124 & 14100 & 1000 \\
\hline $\mathrm{V}$ & 421 & 11 & 33 & 425 & 7 & 98 & 425 & 18 \\
\hline $\mathrm{Cr}$ & 17.0 & 1.3 & 39 & 17 & 2 & 91 & 17 & 2 \\
\hline Mn & 1482 & 34 & 40 & 1463 & 23 & 10 & 1550 & 70 \\
\hline Co & 37.2 & 1.8 & 39 & 38 & 1 & 118 & 38 & 2 \\
\hline $\mathrm{Ni}$ & 12.2 & 0.8 & 29 & 12.7 & 0.9 & 126 & 13 & 2 \\
\hline $\mathrm{Cu}$ & 17.1 & 1.1 & 35 & 18 & 1 & 78 & 21 & 5 \\
\hline $\mathrm{Zn}$ & 146 & 8 & 38 & 153 & 9 & 14 & 125 & 5 \\
\hline $\mathrm{Ga}$ & 22.7 & 1.1 & 38 & 24 & 1 & 71 & 23 & 1 \\
\hline $\mathrm{Rb}$ & 48.5 & 2.3 & 39 & 51 & 3 & 133 & 47 & 0.5 \\
\hline $\mathrm{Sr}$ & 326 & 12 & 39 & 321 & 6 & 122 & 342 & 4 \\
\hline $\mathrm{Y}$ & 31.6 & 5.5 & 38 & 31 & 2 & 138 & 35 & 3 \\
\hline $\mathrm{Zr}$ & 172 & 20 & 32 & 167 & 8 & 127 & 184 & 15 \\
\hline $\mathrm{Nb}$ & 11.1 & 0.8 & 42 & 10.9 & 0.6 & 133 & 12.5 & 1 \\
\hline Mo & 237 & 10 & 42 & n.d & & & 270 & 30 \\
\hline Sn & 2.67 & 0.46 & 36 & 2.4 & 0.4 & 14 & 2.6 & 0.4 \\
\hline $\mathrm{Sb}$ & 0.34 & 0.06 & 25 & 0.51 & 0.87 & 10 & 0.35 & 0.08 \\
\hline Cs & 1.18 & 0.07 & 34 & 1.17 & 0.08 & 71 & 1.16 & 0.07 \\
\hline Ва & 647 & 21 & 34 & 641 & 14 & 125 & 683 & 7 \\
\hline $\mathrm{La}$ & 25.1 & 1.4 & 42 & 25 & 1 & 144 & 24.7 & 0.3 \\
\hline $\mathrm{Ce}$ & 51.5 & 1.8 & 41 & 52 & 2 & 139 & 53.3 & 0.5 \\
\hline $\operatorname{Pr}$ & 6.37 & 0.36 & 41 & 6.3 & 0.4 & 140 & 6.7 & 0.4 \\
\hline $\mathrm{Nd}$ & 27.6 & 2.1 & 42 & 27 & 1 & 132 & 28.9 & 0.3 \\
\hline $\mathrm{Sm}$ & 6.31 & 0.57 & 38 & 6.3 & 0.5 & 115 & 6.59 & 0.07 \\
\hline $\mathrm{Eu}$ & 1.95 & 0.12 & 37 & 1.91 & 0.09 & 108 & 1.97 & 0.02 \\
\hline $\mathrm{Gd}$ & 6.32 & 0.81 & 32 & 6.5 & 0.6 & 112 & 6.71 & 0.07 \\
\hline $\mathrm{Tb}$ & 0.96 & 0.11 & 37 & 0.95 & 0.07 & 115 & 1.02 & 0.08 \\
\hline Dy & 5.92 & 0.73 & 37 & 6 & 0.4 & 106 & 6.44 & 0.06 \\
\hline Но & 1.21 & 0.15 & 35 & 1.2 & 0.07 & 104 & 1.27 & 0.08 \\
\hline Er & 3.29 & 0.45 & 29 & 3.3 & 0.2 & 94 & 3.7 & 0.04 \\
\hline $\mathrm{Tm}$ & 0.47 & 0.07 & 33 & 0.46 & 0.04 & 111 & 0.51 & 0.04 \\
\hline $\mathrm{Yb}$ & 3.38 & 0.28 & 30 & 3.2 & 0.3 & 112 & 3.39 & 0.03 \\
\hline $\mathrm{Lu}$ & 0.48 & 0.06 & 35 & 0.47 & 0.04 & 109 & 0.503 & 0.005 \\
\hline Hf & 4.47 & 0.61 & 36 & 4.5 & 0.4 & 94 & 4.84 & 0.28 \\
\hline Ta & 0.62 & 0.08 & 36 & 0.63 & 0.06 & 97 & 0.78 & 0.06 \\
\hline $\mathrm{Pb}$ & 10.1 & 0.5 & 36 & 10.9 & 0.5 & 104 & 11 & 1 \\
\hline Th & 5.66 & 0.38 & 36 & 5.5 & 0.2 & 103 & 5.9 & 0.3 \\
\hline $\mathrm{U}$ & 1.69 & 0.09 & 35 & 1.7 & 0.08 & 120 & 1.69 & 0.12 \\
\hline
\end{tabular}

${ }^{* *}$ Mean values obtained with various techniques (ID-TIMS, MC-LAICPMS, EPMA, LA-ICPMS). n: number of determinations; Recom: recommended values; n.d: not determined. 
Table 6. Obtained values in $\mathrm{mg} \cdot \mathrm{kg}^{-1}$ for BIR-1G, compared with values quoted in [11] (LA-ICPMS technique) and preferred values in [14].

\begin{tabular}{|c|c|c|c|c|c|c|c|c|}
\hline \multirow{2}{*}{ Element } & \multicolumn{3}{|c|}{ Obtained values } & \multicolumn{3}{|c|}{ Gao et al. [11] } & \multicolumn{2}{|c|}{ GEOREM [14] } \\
\hline & Mean & sd & $\mathrm{n}$ & Mean & sd & $\mathrm{n}$ & Recom $^{* *}$ & $\mathrm{sd}^{* *}$ \\
\hline $\mathrm{Li}$ & 3.28 & 0.36 & 38 & 3.6 & 0.4 & 57 & 3 & 0.7 \\
\hline $\mathrm{Be}$ & 0.44 & 0.15 & 13 & 0.69 & 0.88 & 9 & 0.1 & \\
\hline $\mathrm{Mg}$ & 60101 & 6839 & 38 & 58471 & 1517 & 65 & 56400 & 600 \\
\hline $\mathrm{P}$ & 109 & 6 & 21 & n.d & & & 118 & 13 \\
\hline Sc & 38.9 & 2.6 & 44 & 41 & 1 & 95 & 43 & 3 \\
\hline $\mathrm{Ti}$ & 5786 & 598 & 37 & 5532 & 323 & 97 & 5400 & 200 \\
\hline $\mathrm{V}$ & 330 & 11 & 39 & 338 & 6 & 91 & 326 & 32 \\
\hline $\mathrm{Cr}$ & 394 & 13 & 40 & 403 & 11 & 85 & 392 & 24 \\
\hline Mn & 1341 & 56 & 44 & 1417 & 30 & 17 & 1472 & 77 \\
\hline Co & 54.2 & 2.0 & 37 & 57 & 1 & 78 & 52 & 5 \\
\hline $\mathrm{Ni}$ & 176 & 8 & 40 & 190 & 6 & 85 & 178 & 18 \\
\hline $\mathrm{Cu}$ & 124 & 4 & 35 & 132 & 6 & 69 & 119 & 12 \\
\hline $\mathrm{Zn}$ & 77.6 & 4.9 & 39 & 86 & 5 & 25 & 78 & 17 \\
\hline $\mathrm{Ga}$ & 16.0 & 0.7 & 37 & 17 & 1 & 76 & 15 & 2 \\
\hline $\mathrm{Rb}$ & 0.22 & 0.03 & 33 & 0.26 & 0.05 & 68 & 0.197 & 0.007 \\
\hline $\mathrm{Sr}$ & 106 & 4 & 41 & 104 & 2 & 102 & 109 & 2 \\
\hline $\mathrm{Y}$ & 12.2 & 1.0 & 36 & 13.3 & 0.6 & 99 & 14.3 & 1.4 \\
\hline $\mathrm{Zr}$ & 12.0 & 1.2 & 36 & 12.9 & 0.6 & 98 & 14 & 1.2 \\
\hline $\mathrm{Nb}$ & 0.51 & 0.05 & 40 & 0.48 & 0.04 & 94 & 0.52 & 0.04 \\
\hline Mo & 0.089 & 0.03 & 23 & n.d & & & 0.075 & 0.011 \\
\hline Sn & 1.27 & 0.26 & 41 & 0.84 & 0.23 & 9 & 2.3 & 1.3 \\
\hline $\mathrm{Sb}$ & 0.55 & 0.15 & 39 & 0.47 & 0.13 & 12 & 0.56 & 0.09 \\
\hline Cs & 0.011 & 0.004 & 21 & 0.0069 & 0.0020 & 31 & 0.007 & 0.002 \\
\hline $\mathrm{Ba}$ & 6.31 & 0.44 & 40 & 6.3 & 0.3 & 99 & 6.5 & 0.07 \\
\hline $\mathrm{La}$ & 0.59 & 0.05 & 43 & 0.60 & 0.04 & 91 & 0.609 & 0.02 \\
\hline $\mathrm{Ce}$ & 1.92 & 0.12 & 45 & 1.9 & 0.08 & 76 & 1.89 & 0.04 \\
\hline $\operatorname{Pr}$ & 0.36 & 0.04 & 46 & 0.36 & 0.02 & 78 & 0.37 & 0.02 \\
\hline $\mathrm{Nd}$ & 2.29 & 0.20 & 42 & 2.3 & 0.2 & 73 & 2.37 & 0.03 \\
\hline $\mathrm{Sm}$ & 1.06 & 0.11 & 37 & 1.1 & 0.1 & 72 & 1.09 & 0.02 \\
\hline $\mathrm{Eu}$ & 0.52 & 0.05 & 39 & 0.51 & 0.04 & 78 & 0.517 & 0.005 \\
\hline Gd & 1.59 & 0.17 & 33 & 1.6 & 0.1 & 76 & 1.85 & 0.02 \\
\hline $\mathrm{Tb}$ & 0.31 & 0.04 & 30 & 0.32 & 0.03 & 81 & 0.35 & 0.04 \\
\hline Dy & 2.19 & 0.21 & 32 & 2.3 & 0.2 & 78 & 2.55 & 0.02 \\
\hline Но & 0.50 & 0.06 & 30 & 0.51 & 0.05 & 86 & 0.56 & 0.03 \\
\hline Er & 1.44 & 0.10 & 26 & 1.5 & 0.1 & 80 & 1.7 & 0.02 \\
\hline $\mathrm{Tm}$ & 0.21 & 0.02 & 35 & 0.22 & 0.02 & 69 & 0.24 & 0.03 \\
\hline $\mathrm{Yb}$ & 1.46 & 0.17 & 34 & 1.5 & 0.1 & 80 & 1.64 & 0.03 \\
\hline $\mathrm{Lu}$ & 0.22 & 0.02 & 27 & 0.23 & 0.02 & 63 & 0.248 & 0.009 \\
\hline $\mathrm{Hf}$ & 0.49 & 0.10 & 28 & 0.53 & 0.06 & 71 & 0.57 & 0.03 \\
\hline Ta & 0.03 & 0.01 & 40 & 0.032 & 0.01 & 80 & 0.036 & 0.006 \\
\hline $\mathrm{Pb}$ & 3.36 & 0.21 & 31 & 3.6 & 0.2 & 78 & 3.7 & 0.3 \\
\hline Th & 0.03 & 0.01 & 44 & 0.028 & 0.01 & 65 & 0.03 & 0.002 \\
\hline $\mathrm{U}$ & 0.02 & 0.01 & 31 & 0.032 & 0.01 & 67 & 0.023 & 0.006 \\
\hline
\end{tabular}

${ }^{* *}$ Mean values obtained with various techniques (ID-TIMS, MC-LAICPMS, EPMA, LA-ICPMS). n: number of determinations; Recom: recommended values; n.d: not determined. 
Table 7. Obtained values in $\mathrm{mg} \cdot \mathrm{kg}^{-1}$ for BHVO-2G, compared with values quoted in [11] (LA-ICPMS technique) and preferred values in [14].

\begin{tabular}{|c|c|c|c|c|c|c|c|c|}
\hline \multirow{2}{*}{ Element } & \multicolumn{3}{|c|}{ Obtained values } & \multicolumn{3}{|c|}{ Gao et al. [11] } & \multicolumn{2}{|c|}{ GEOREM [14] } \\
\hline & Mean & sd & $\mathrm{n}$ & Mean & sd & $\mathrm{n}$ & Recom $^{* *}$ & $\mathrm{sd}^{* *}$ \\
\hline $\mathrm{Li}$ & 4.67 & 0.42 & 65 & 5.0 & 0.4 & 26 & 4.4 & 0.8 \\
\hline $\mathrm{Be}$ & 1.24 & 0.36 & 40 & 1.4 & 0.2 & 6 & 1.3 & 0.2 \\
\hline $\mathrm{Mg}$ & 45594 & 4910 & 51 & 42682 & 1071 & 25 & 42992 & 121 \\
\hline $\mathrm{P}$ & 1281 & 126 & 40 & & & & 1266 & 87 \\
\hline Sc & 29.6 & 2.1 & 72 & 31 & 1 & 51 & 33 & 2 \\
\hline $\mathrm{Ti}$ & 16165 & 1249 & 39 & 15621 & 453 & 53 & 16300 & 900 \\
\hline V & 319 & 9 & 64 & 329 & 9 & 42 & 308 & 19 \\
\hline $\mathrm{Cr}$ & 288 & 9 & 67 & 285 & 14 & 51 & 293 & 12 \\
\hline $\mathrm{Mn}$ & 1310 & 58 & 74 & 1345 & 25 & 22 & 1317 & 233 \\
\hline Co & 45.2 & 2.1 & 70 & 47 & 2 & 53 & 44 & 2 \\
\hline $\mathrm{Ni}$ & 120 & 7 & 70 & 112 & 9 & 48 & 116 & 7 \\
\hline $\mathrm{Cu}$ & 120 & 7 & 72 & 142 & 10 & 52 & 127 & 11 \\
\hline $\mathrm{Zn}$ & 114 & 6 & 72 & 107 & 26 & 36 & 102 & 6 \\
\hline $\mathrm{Ga}$ & 21.7 & 0.8 & 71 & 21 & 1 & 44 & 22 & 3 \\
\hline $\mathrm{Rb}$ & 9.43 & 0.53 & 69 & 10.1 & 0.6 & 49 & 9.2 & 0.04 \\
\hline $\mathrm{Sr}$ & 388 & 16 & 72 & 328 & 10 & 53 & 396 & 1 \\
\hline $\mathrm{Y}$ & 22.1 & 1.6 & 45 & 23 & 1 & 57 & 26 & 2 \\
\hline $\mathrm{Zr}$ & 155 & 11 & 49 & 160 & 8 & 56 & 170 & 7 \\
\hline $\mathrm{Nb}$ & 16.9 & 1.2 & 55 & 16.4 & 0.7 & 56 & 18.3 & 0.8 \\
\hline Mo & 4.09 & 0.29 & 62 & n.d & & & 3.8 & 0.2 \\
\hline Sn & 2.06 & 0.17 & 58 & 2.6 & 0.6 & 22 & 2.6 & 0.6 \\
\hline $\mathrm{Sb}$ & 0.24 & 0.07 & 35 & 0.21 & 0.04 & 10 & 0.3 & 0.13 \\
\hline Cs & 0.10 & 0.01 & 65 & 0.11 & 0.02 & 29 & 0.1 & 0.02 \\
\hline $\mathrm{Ba}$ & 129 & 7 & 71 & 128 & 4 & 56 & 131 & 2 \\
\hline $\mathrm{La}$ & 15.2 & 0.9 & 63 & 15.6 & 0.6 & 38 & 15.2 & 0.2 \\
\hline $\mathrm{Ce}$ & 37.5 & 1.4 & 71 & 37 & 1 & 32 & 37.6 & 0.2 \\
\hline $\operatorname{Pr}$ & 5.08 & 0.22 & 68 & 5.0 & 0.3 & 33 & 5.35 & 0.22 \\
\hline $\mathrm{Nd}$ & 23.5 & 1.3 & 67 & 24 & 1 & 32 & 24.5 & 0.2 \\
\hline $\mathrm{Sm}$ & 5.75 & 0.35 & 59 & 5.8 & 0.5 & 32 & 6.1 & 0.03 \\
\hline $\mathrm{Eu}$ & 2.01 & 0.11 & 67 & 2.0 & 0.1 & 28 & 2.07 & 0.01 \\
\hline Gd & 5.50 & 0.34 & 51 & 5.9 & 0.4 & 30 & 6.16 & 0.05 \\
\hline $\mathrm{Tb}$ & 0.85 & 0.06 & 40 & 0.86 & 0.06 & 31 & 0.92 & 0.04 \\
\hline Dy & 4.92 & 0.38 & 46 & 4.9 & 0.4 & 33 & 5.28 & 0.05 \\
\hline Но & 0.91 & 0.07 & 45 & 0.91 & 0.06 & 32 & 0.98 & 0.04 \\
\hline Er & 2.28 & 0.18 & 39 & 2.3 & 0.2 & 28 & 2.56 & 0.02 \\
\hline $\mathrm{Tm}$ & 0.29 & 0.04 & 56 & 0.30 & 0.05 & 32 & 0.34 & 0.02 \\
\hline $\mathrm{Yb}$ & 1.96 & 0.17 & 41 & 2.0 & 0.2 & 30 & 2.01 & 0.02 \\
\hline $\mathrm{Lu}$ & 0.25 & 0.03 & 51 & 0.26 & 0.04 & 29 & 0.279 & 0.003 \\
\hline $\mathrm{Hf}$ & 4.02 & 0.31 & 43 & 4.1 & 0.4 & 52 & 4.32 & 0.18 \\
\hline Ta & 0.96 & 0.08 & 54 & 0.94 & 0.07 & 54 & 1.15 & 0.1 \\
\hline $\mathrm{Pb}$ & 1.80 & 0.13 & 63 & 1.4 & 0.2 & 43 & 1.7 & 0.2 \\
\hline Th & 1.15 & 0.08 & 53 & 1.18 & 0.09 & 48 & 1.22 & 0.05 \\
\hline $\mathrm{U}$ & 0.45 & 0.04 & 56 & 0.44 & 0.03 & 42 & 0.403 & 0.003 \\
\hline
\end{tabular}

${ }^{* *}$ Mean values obtained with various techniques (ID-TIMS, MC-LAICPMS, EPMA, LA-ICPMS). n: number of determinations; Recom: recommended values; n.d: not determined. 
Table 8. Obtained values in $\mathrm{mg} \cdot \mathrm{kg}^{-1}$ for 12 determinations of basalt glass $\mathrm{KL}-2 \mathrm{G}$, compared with values quoted in [15].

\begin{tabular}{|c|c|c|c|c|c|c|}
\hline \multirow{2}{*}{ Elements } & \multicolumn{3}{|c|}{ Obtained values } & \multicolumn{3}{|c|}{ Jochum et al. [15] } \\
\hline & Mean & sd & \% rsd & Mean & $\mathrm{U}$ & $\%$ var. \\
\hline $\mathrm{Li}$ & 5.35 & 0.48 & 8.9 & 5.10 & 0.50 & 9.8 \\
\hline Sc & 29.7 & 1.2 & 4.0 & 31.8 & 0.90 & 2.8 \\
\hline $\mathrm{Ti}$ & 16079 & 1279 & 8.0 & 15347 & 540 & 3.5 \\
\hline V & 294 & 14 & 4.7 & 309 & 38 & 12.3 \\
\hline $\mathrm{Cr}$ & 283 & 6 & 2.1 & 294 & 27 & 9.2 \\
\hline Mn & 1190 & 53 & 4.5 & 1278 & 70 & 5.5 \\
\hline Co & 39.4 & 1.1 & 2.7 & 41.2 & 2.3 & 5.6 \\
\hline $\mathrm{Ni}$ & 101 & 5.0 & 4.9 & 112 & 5 & 4.5 \\
\hline $\mathrm{Cu}$ & 81.6 & 3.5 & 4.2 & 87.9 & 9.1 & 10.4 \\
\hline $\mathrm{Zn}$ & 105 & 4 & 3.4 & 110 & 10 & 9.1 \\
\hline $\mathrm{Ga}$ & 20.1 & 0.5 & 2.5 & 20.0 & 1.2 & 6.0 \\
\hline $\mathrm{Rb}$ & 8.38 & 0.77 & 9.1 & 8.70 & 0.40 & 4.6 \\
\hline $\mathrm{Sr}$ & 342 & 11 & 3.0 & 356 & 8 & 2.2 \\
\hline $\mathrm{Y}$ & 22.2 & 0.5 & 2.4 & 25.4 & 1.1 & 4.3 \\
\hline $\mathrm{Zr}$ & 129 & 3 & 2.0 & 152 & 5 & 3.3 \\
\hline $\mathrm{Nb}$ & 14.2 & 0.3 & 1.9 & 15.0 & 0.5 & 3.3 \\
\hline Mo & 2.98 & 0.14 & 4.7 & 3.6 & 0.6 & 16.7 \\
\hline Sn & 1.51 & 0.12 & 8.3 & 1.54 & 0.29 & 18.8 \\
\hline $\mathrm{Sb}$ & 0.126 & 0.016 & 12.8 & 0.14 & 0.03 & 21.4 \\
\hline Cs & 0.108 & 0.009 & 8.0 & 0.115 & 0.009 & 7.8 \\
\hline $\mathrm{Ba}$ & 110 & 3 & 2.0 & 123 & 5 & 4.1 \\
\hline $\mathrm{La}$ & 12.9 & 0.4 & 3.4 & 13.1 & 0.2 & 1.5 \\
\hline $\mathrm{Ce}$ & 31.3 & 1.7 & 5.5 & 32.4 & 0.7 & 2.2 \\
\hline $\operatorname{Pr}$ & 4.34 & 0.14 & 3.1 & 4.60 & 0.10 & 2.2 \\
\hline Nd & 20.8 & 0.6 & 2.7 & 21.6 & 0.4 & 1.9 \\
\hline $\mathrm{Sm}$ & 5.30 & 0.20 & 3.8 & 5.54 & 0.09 & 1.6 \\
\hline $\mathrm{Eu}$ & 1.91 & 0.09 & 4.7 & 1.92 & 0.04 & 2.1 \\
\hline Gd & 5.29 & 0.32 & 3.8 & 5.92 & 0.20 & 3.4 \\
\hline $\mathrm{Tb}$ & 0.79 & 0.04 & 5.0 & 0.890 & 0.031 & 3.5 \\
\hline Dy & 4.78 & 0.35 & 6.6 & 5.22 & 0.12 & 2.3 \\
\hline Ho & 0.88 & 0.05 & 5.6 & 0.961 & 0.022 & 2.3 \\
\hline Er & 2.26 & 0.10 & 4.3 & 2.54 & 0.07 & 2.8 \\
\hline $\mathrm{Tm}$ & 0.30 & 0.01 & 4.5 & 0.331 & 0.001 & 0.3 \\
\hline $\mathrm{Yb}$ & 1.95 & 0.12 & 5.9 & 2.10 & 0.05 & 2.4 \\
\hline $\mathrm{Lu}$ & 0.26 & 0.01 & 4.6 & 0.285 & 0.009 & 3.2 \\
\hline $\mathrm{Hf}$ & 3.53 & 0.16 & 4.6 & 3.93 & 0.14 & 3.6 \\
\hline Тa & 0.88 & 0.05 & 6.2 & 0.961 & 0.022 & 2.3 \\
\hline $\mathrm{Pb}$ & 1.94 & 0.06 & 2.9 & 2.07 & 0.10 & 4.8 \\
\hline Th & 0.94 & 0.04 & 4.3 & 1.02 & 0.03 & 2.9 \\
\hline $\mathrm{U}$ & 0.53 & 0.08 & 14.5 & 0.548 & 0.016 & 2.9 \\
\hline
\end{tabular}

U: Uncertainty at 95\% confidence level; \% var.: percent of variability calculated. 
Table 9. Mean obtained value for 12 determinations in $\mathrm{mg} \cdot \mathrm{kg}^{-1}$ for basalt glass ML-3BG, compared with values quoted in [15].

\begin{tabular}{|c|c|c|c|c|c|c|}
\hline \multirow{2}{*}{ Elements } & \multicolumn{3}{|c|}{ Obtained values } & \multicolumn{3}{|c|}{ Jochum et al. [15] } \\
\hline & Mean & sd & $\%$ rsd & Mean & $\mathrm{U}$ & $\%$ var. \\
\hline $\mathrm{Li}$ & 4.25 & 0.15 & 3.6 & 4.5 & 0.4 & 8.9 \\
\hline Sc & 28.8 & 1.2 & 4.3 & 31.6 & 2.9 & 9.2 \\
\hline $\mathrm{Ti}$ & 13198 & 836 & 6.3 & 12767 & 1259 & 9.9 \\
\hline V & 264 & 6.9 & 2.6 & 268 & 23 & 10.5 \\
\hline $\mathrm{Cr}$ & 154 & 4 & 2.9 & 179 & 44 & 24.6 \\
\hline $\mathrm{Mn}$ & 1193 & 42 & 3.5 & 1224 & 108 & 8.9 \\
\hline Co & 40.2 & 1.5 & 3.8 & 42.0 & 6.0 & 14.3 \\
\hline $\mathrm{Ni}$ & 97.2 & 2.5 & 2.6 & 103 & 9 & 8.7 \\
\hline $\mathrm{Cu}$ & 107 & 3 & 2.5 & 110 & 9 & 8.2 \\
\hline $\mathrm{Zn}$ & 110 & 5 & 4.5 & 102 & 21 & 20.6 \\
\hline $\mathrm{Ga}$ & 19.0 & 0.4 & 2.1 & 18.5 & 2.8 & 15.1 \\
\hline $\mathrm{Rb}$ & 5.68 & 0.25 & 4.3 & 5.82 & 0.6 & 10.3 \\
\hline $\mathrm{Sr}$ & 308 & 12 & 3.9 & 309 & 12 & 3.9 \\
\hline $\mathrm{Y}$ & 21.1 & 1.2 & 5.6 & 23.3 & 1.7 & 7.3 \\
\hline $\mathrm{Zr}$ & 105 & 6 & 5.3 & 117 & 8 & 6.8 \\
\hline $\mathrm{Nb}$ & 7.99 & 0.34 & 4.2 & 8.43 & 0.38 & 4.5 \\
\hline Mo & 15.5 & 0.9 & 5.8 & 16.1 & 3.4 & 21.1 \\
\hline Sn & 1.17 & 0.08 & 6.6 & 1.15 & 0.48 & 41.7 \\
\hline $\mathrm{Sb}$ & 0.123 & 0.015 & 12.2 & 0.11 & 0.04 & 36.4 \\
\hline Cs & 0.131 & 0.011 & 8.5 & 0.137 & 0.027 & 19.7 \\
\hline $\mathrm{Ba}$ & 74.5 & 2.5 & 3.3 & 79.2 & 4.1 & 5.2 \\
\hline $\mathrm{La}$ & 8.84 & 0.30 & 3.4 & 9.04 & 0.45 & 5.0 \\
\hline Ce & 22.5 & 0.8 & 3.4 & 23.2 & 0.9 & 3.9 \\
\hline $\operatorname{Pr}$ & 3.23 & 0.15 & 4.5 & 3.42 & 0.14 & 4.1 \\
\hline $\mathrm{Nd}$ & 16.2 & 0.5 & 2.8 & 16.9 & 0.6 & 3.6 \\
\hline $\mathrm{Sm}$ & 4.58 & 0.18 & 3.9 & 4.74 & 0.24 & 5.1 \\
\hline $\mathrm{Eu}$ & 1.62 & 0.09 & 5.7 & 1.67 & 0.06 & 3.6 \\
\hline Gd & 4.65 & 0.35 & 7.4 & 5.10 & 0.32 & 6.3 \\
\hline $\mathrm{Tb}$ & 0.72 & 0.03 & 4.9 & 0.783 & 0.057 & 7.3 \\
\hline Dy & 4.45 & 0.39 & 8.8 & 4.84 & 0.21 & 4.3 \\
\hline Но & 0.85 & 0.05 & 6.2 & 0.901 & 0.051 & 5.7 \\
\hline Er & 2.21 & 0.16 & 7.1 & 2.41 & 0.13 & 5.4 \\
\hline $\mathrm{Tm}$ & 0.30 & 0.02 & 7.1 & 0.324 & 0.02 & 6.2 \\
\hline $\mathrm{Yb}$ & 1.97 & 0.10 & 5.2 & 2.06 & 0.12 & 5.8 \\
\hline $\mathrm{Lu}$ & 0.27 & 0.023 & 8.7 & 0.287 & 0.019 & 6.6 \\
\hline Hf & 2.97 & 0.24 & 8.2 & 3.14 & 0.20 & 6.4 \\
\hline Ta & 0.52 & 0.04 & 7.0 & 0.552 & 0.033 & 6.0 \\
\hline $\mathrm{Pb}$ & 1.30 & 0.07 & 5.5 & 1.40 & 0.15 & 10.7 \\
\hline Th & 0.51 & 0.03 & 5.0 & 0.55 & 0.03 & 5.5 \\
\hline $\mathrm{U}$ & 0.41 & 0.020 & 4.9 & 0.448 & 0.055 & 12.3 \\
\hline
\end{tabular}

U: Uncertainty at 95\% confidence level; \% var.: percent of variability calculated. 
Table 10. Mean obtained values for 10 determinations in $\mathrm{mg} \cdot \mathrm{kg}^{-1}$ for andesite glass StHs6/80G, compared with values quoted in [15].

\begin{tabular}{|c|c|c|c|c|c|c|}
\hline \multirow{2}{*}{ Elements } & \multicolumn{3}{|c|}{ Obtained values } & \multicolumn{3}{|c|}{ Jochum et al. [15] } \\
\hline & Mean & sd & \% rsd & Mean & $\mathrm{U}$ & $\%$ var \\
\hline $\mathrm{Li}$ & 18.0 & 1.11 & 6.2 & 21.7 & 3.8 & 17.5 \\
\hline Sc & 10.4 & 0.46 & 4.4 & 11.9 & 1.3 & 10.9 \\
\hline $\mathrm{Ti}$ & 4887 & 103 & 2.1 & 5591 & 487 & 8.71 \\
\hline $\mathrm{V}$ & 76.5 & 2.95 & 3.9 & 88.0 & 8.0 & 9.10 \\
\hline $\mathrm{Cr}$ & 12.1 & 0.53 & 4.4 & 15.5 & 3.4 & 21.9 \\
\hline Mn & 528 & 17 & 3.3 & 558 & 31 & 5.56 \\
\hline Co & 10.9 & 0.55 & 5.0 & 13.6 & 1.6 & 11.8 \\
\hline $\mathrm{Ni}$ & 15.7 & 0.57 & 3.6 & 23.4 & 7.1 & 30.3 \\
\hline $\mathrm{Cu}$ & 29.5 & 1.66 & 5.6 & 42.0 & 13.4 & 31.9 \\
\hline $\mathrm{Zn}$ & 60.8 & 4.23 & 7.0 & 71.0 & 10.0 & 14.1 \\
\hline $\mathrm{Ga}$ & 18.0 & 0.72 & 4.0 & 22.0 & 5.10 & 23.2 \\
\hline $\mathrm{Rb}$ & 24.1 & 0.94 & 3.9 & 31.7 & 4.40 & 13.9 \\
\hline $\mathrm{Sr}$ & 462 & 17.2 & 3.6 & 477 & 21.0 & 4.42 \\
\hline $\mathrm{Y}$ & 10.8 & 0.64 & 5.9 & 11.4 & 1.10 & 9.65 \\
\hline $\mathrm{Zr}$ & 105 & 6.00 & 5.7 & 115 & 10.0 & 8.70 \\
\hline $\mathrm{Nb}$ & 6.31 & 0.29 & 4.6 & 6.90 & 0.55 & 7.97 \\
\hline Mo & 1.42 & 0.10 & 7.3 & 1.80 & 0.80 & 44.4 \\
\hline Sn & 1.05 & 0.16 & 15.5 & 1.10 & 0.30 & 27.3 \\
\hline $\mathrm{Sb}$ & 0.20 & 0.04 & 21.3 & 0.18 & 0.09 & 50.0 \\
\hline Cs & 1.29 & 0.03 & 2.3 & 1.75 & 0.23 & 13.1 \\
\hline $\mathrm{Ba}$ & 277 & 6.28 & 2.3 & 298 & 15.0 & 5.0 \\
\hline $\mathrm{La}$ & 11.5 & 0.42 & 3.6 & 12.1 & 0.70 & 5.8 \\
\hline Ce & 23.8 & 0.74 & 3.1 & 26.3 & 1.20 & 4.6 \\
\hline $\operatorname{Pr}$ & 2.97 & 0.11 & 3.8 & 3.21 & 0.16 & 5.0 \\
\hline $\mathrm{Nd}$ & 12.7 & 0.35 & 2.8 & 13.0 & 0.60 & 4.6 \\
\hline $\mathrm{Sm}$ & 2.59 & 0.13 & 5.2 & 2.79 & 0.12 & 4.3 \\
\hline $\mathrm{Eu}$ & 0.95 & 0.03 & 2.7 & 0.96 & 0.05 & 4.8 \\
\hline $\mathrm{Gd}$ & 2.56 & 0.18 & 7.0 & 2.55 & 0.20 & 7.8 \\
\hline $\mathrm{Tb}$ & 0.33 & 0.02 & 4.8 & 0.37 & 0.02 & 6.3 \\
\hline Dy & 2.09 & 0.13 & 6.1 & 2.22 & 0.15 & 6.8 \\
\hline Ho & 0.40 & 0.03 & 7.7 & 0.42 & 0.03 & 6.7 \\
\hline Er & 1.09 & 0.03 & 3.1 & 1.19 & 0.08 & 6.7 \\
\hline $\mathrm{Tm}$ & 0.16 & 0.02 & 10.6 & 0.17 & 0.02 & 8.8 \\
\hline $\mathrm{Yb}$ & 1.10 & 0.05 & 4.6 & 1.14 & 0.08 & 7.0 \\
\hline $\mathrm{Lu}$ & 0.16 & 0.02 & 9.8 & 0.17 & 0.02 & 10.2 \\
\hline $\mathrm{Hf}$ & 2.78 & 0.18 & 6.4 & 2.98 & 0.21 & 7.0 \\
\hline Ta & 0.39 & 0.01 & 3.9 & 0.42 & 0.04 & 8.5 \\
\hline $\mathrm{Pb}$ & 10.4 & 0.50 & 4.8 & 10.4 & 1.97 & 18.9 \\
\hline Th & 2.18 & 0.08 & 3.5 & 2.30 & 0.21 & 9.1 \\
\hline $\mathrm{U}$ & 0.91 & 0.04 & 4.5 & 1.02 & 0.11 & 10.8 \\
\hline
\end{tabular}

U: Uncertainty at 95\% confidence level; \% var.: percent of variability calculated. 
Table 11. Mean obtained values for 6 determinations in $\mathrm{mg} \cdot \mathrm{kg}^{-1}$ for quartz diorite glass T-1G, compared with values quoted in [15].

\begin{tabular}{|c|c|c|c|c|c|c|}
\hline \multirow{2}{*}{ Elements } & \multicolumn{3}{|c|}{ Obtained values } & \multicolumn{3}{|c|}{ Jochum et al. [15]. } \\
\hline & Mean & sd & $\%$ rsd & Mean & $\mathrm{U}$ & $\%$ var \\
\hline $\mathrm{Li}$ & 19.7 & 0.4 & 2.0 & 20.2 & 1.6 & 7.9 \\
\hline Sc & 30.12 & 0.37 & 1.2 & 27.1 & 2.4 & 8.9 \\
\hline $\mathrm{Ti}$ & 5255 & 83 & 1.6 & 4699 & 210 & 4.5 \\
\hline $\mathrm{V}$ & 167 & 3 & 2.1 & 190 & 16 & 8.4 \\
\hline $\mathrm{Cr}$ & 15.7 & 0.6 & 3.9 & 20.3 & 1.5 & 7.4 \\
\hline $\mathrm{Mn}$ & 942 & 15 & 1.6 & 968 & 93 & 9.6 \\
\hline Co & 17.0 & 0.3 & 2.0 & 19.1 & 1.8 & 9.4 \\
\hline $\mathrm{Ni}$ & 8.38 & 0.22 & 2.6 & 10.7 & 2.4 & 22.4 \\
\hline $\mathrm{Cu}$ & 16.4 & 0.3 & 1.9 & 18.5 & 2.2 & 11.9 \\
\hline $\mathrm{Zn}$ & 60.3 & 2.2 & 3.6 & 69 & 12 & 17.4 \\
\hline Ga & 18.3 & 0.2 & 1.3 & 19.8 & 1.5 & 7.6 \\
\hline $\mathrm{Rb}$ & 74.7 & 1.6 & 2.1 & 80.5 & 9 & 11.2 \\
\hline $\mathrm{Sr}$ & 282 & 5 & 1.8 & 283 & 16 & 5.7 \\
\hline $\mathrm{Y}$ & 28.2 & 0.3 & 1.2 & 24.1 & 1.8 & 7.5 \\
\hline $\mathrm{Zr}$ & 169 & 2 & 1.3 & 141 & 12 & 8.5 \\
\hline $\mathrm{Nb}$ & 8.41 & 0.15 & 1.8 & 8.92 & 0.53 & 5.9 \\
\hline Mo & 5.60 & 0.19 & 3.3 & 3.6 & 2.5 & 69.4 \\
\hline Sn & 1.70 & 0.15 & 8.7 & 1.8 & 0.6 & 33.3 \\
\hline $\mathrm{Sb}$ & 0.26 & 0.03 & 10.8 & 0.23 & 0.08 & 34.8 \\
\hline Cs & 2.59 & 0.08 & 3.1 & 2.58 & 0.39 & 15.1 \\
\hline $\mathrm{Ba}$ & 385 & 8 & 2.1 & 393 & 23 & 5.9 \\
\hline $\mathrm{La}$ & 78.0 & 1.6 & 2.0 & 72.1 & 4.7 & 6.5 \\
\hline Ce & 121 & 2 & 1.9 & 129 & 6 & 4.7 \\
\hline Pr & 12.8 & 0.2 & 1.7 & 12.5 & 0.8 & 6.4 \\
\hline $\mathrm{Nd}$ & 46.3 & 0.8 & 1.8 & 42 & 2.5 & 6.0 \\
\hline $\mathrm{Sm}$ & 7.31 & 0.20 & 2.8 & 6.58 & 0.33 & 5.0 \\
\hline $\mathrm{Eu}$ & 1.22 & 0.05 & 4.0 & 1.20 & 0.07 & 5.8 \\
\hline Gd & 8.41 & 0.49 & 5.8 & 5.32 & 0.67 & 12.6 \\
\hline $\mathrm{Tb}$ & 0.85 & 0.02 & 2.7 & 0.74 & 0.044 & 5.9 \\
\hline Dy & 5.24 & 0.15 & 2.9 & 4.49 & 0.30 & 6.7 \\
\hline Ho & 1.06 & 0.02 & 2.3 & 0.867 & 0.067 & 7.7 \\
\hline Er & 2.98 & 0.13 & 4.4 & 2.51 & 0.15 & 6.0 \\
\hline $\mathrm{Tm}$ & 0.43 & 0.03 & 8.2 & 0.352 & 0.032 & 9.1 \\
\hline $\mathrm{Yb}$ & 2.89 & 0.13 & 4.5 & 2.39 & 0.24 & 10.0 \\
\hline $\mathrm{Lu}$ & 0.44 & 0.01 & 3.2 & 0.353 & 0.035 & 9.9 \\
\hline $\mathrm{Hf}$ & 4.44 & 0.06 & 1.4 & 3.80 & 0.33 & 8.7 \\
\hline Ta & 0.54 & 0.03 & 4.9 & 0.464 & 0.038 & 8.2 \\
\hline $\mathrm{Pb}$ & 8.29 & 0.22 & 2.7 & 12 & 3.1 & 25.8 \\
\hline Th & 36.0 & 0.5 & 1.5 & 31.2 & 2.7 & 8.7 \\
\hline $\mathrm{U}$ & 1.44 & 0.04 & 3.0 & 1.72 & 0.26 & 15.1 \\
\hline
\end{tabular}

U: Uncertainty at 95\% confidence level; \% var.: percent of variability calculated. 
Table 12. Mean obtained values for 6 determinations in $\mathrm{mg} \cdot \mathrm{kg}^{-1}$ for komatiite GOR-128G, compared with values quoted in [15].

\begin{tabular}{|c|c|c|c|c|c|c|}
\hline \multirow{2}{*}{ Elements } & \multicolumn{3}{|c|}{ Obtained values } & \multicolumn{3}{|c|}{ Jochum et al. [15] } \\
\hline & Mean & sd & $\%$ rsd & Mean & $\mathrm{U}$ & $\%$ var. \\
\hline $\mathrm{Li}$ & 8.33 & 0.44 & 5.3 & 12.2 & 1.5 & 12.3 \\
\hline Sc & 35.6 & 1.5 & 4.3 & 32.5 & 1.8 & 5.5 \\
\hline $\mathrm{Ti}$ & 1865 & 75 & 4.0 & 1811 & 192 & 10.6 \\
\hline $\mathrm{V}$ & 138 & 4 & 2.6 & 191 & 17 & 8.9 \\
\hline $\mathrm{Cr}$ & 1868 & 53 & 2.9 & 2364 & 222 & 9.4 \\
\hline Mn & 1111 & 14 & 1.2 & 1416 & 232 & 16.4 \\
\hline Co & 73.2 & 1.1 & 1.4 & 95.7 & 9.8 & 10.2 \\
\hline $\mathrm{Ni}$ & 840 & 32 & 3.8 & 1076 & 106 & 9.9 \\
\hline $\mathrm{Cu}$ & 48.3 & 4.2 & 8.6 & 64.5 & 17.3 & 26.8 \\
\hline $\mathrm{Zn}$ & 56.2 & 3.6 & 6.5 & 75.0 & 4.6 & 6.1 \\
\hline $\mathrm{Ga}$ & 7.68 & 0.29 & 3.8 & 9.04 & 1.11 & 12.3 \\
\hline $\mathrm{Rb}$ & 0.27 & 0.03 & 10.7 & 0.40 & 0.04 & 10.9 \\
\hline $\mathrm{Sr}$ & 29.2 & 1.3 & 4.3 & 29.5 & 1.9 & 6.4 \\
\hline $\mathrm{Y}$ & 12.5 & 0.3 & 2.4 & 11.8 & 1.2 & 10.2 \\
\hline $\mathrm{Zr}$ & 10.5 & 0.4 & 3.8 & 9.8 & 1.1 & 11.2 \\
\hline $\mathrm{Nb}$ & 0.091 & 0.005 & 5.9 & 0.096 & 0.012 & 12.5 \\
\hline Mo & 0.43 & 0.06 & 14.2 & 0.73 & 0.27 & 37.0 \\
\hline Sn & 0.35 & 0.05 & 15.4 & 0.23 & 0.10 & 42.7 \\
\hline $\mathrm{Sb}$ & $<0.03$ & & & 0.006 & 0.002 & 33.3 \\
\hline Cs & 0.16 & 0.02 & 11.6 & 0.24 & 0.05 & 21.9 \\
\hline $\mathrm{Ba}$ & 0.94 & 0.09 & 10.0 & 1.06 & 0.07 & 6.6 \\
\hline $\mathrm{La}$ & 0.118 & 0.019 & 15.8 & 0.118 & 0.007 & 5.9 \\
\hline $\mathrm{Ce}$ & 0.38 & 0.03 & 9.0 & 0.45 & 0.03 & 6.3 \\
\hline $\operatorname{Pr}$ & 0.091 & 0.015 & 15.9 & 0.098 & 0.005 & 5.1 \\
\hline $\mathrm{Nd}$ & 0.76 & 0.10 & 13.6 & 0.78 & 0.10 & 12.9 \\
\hline $\mathrm{Sm}$ & 0.51 & 0.07 & 14.4 & 0.51 & 0.04 & 7.6 \\
\hline $\mathrm{Eu}$ & 0.25 & 0.04 & 16.9 & 0.26 & 0.02 & 6.1 \\
\hline Gd & 1.19 & 0.10 & 8.4 & 1.15 & 0.11 & 9.6 \\
\hline $\mathrm{Tb}$ & 0.25 & 0.02 & 8.7 & 0.25 & 0.03 & 10.1 \\
\hline Dy & 1.96 & 0.13 & 6.7 & 1.97 & 0.18 & 9.1 \\
\hline Но & 0.45 & 0.03 & 7.6 & 0.44 & 0.04 & 9.9 \\
\hline Er & 1.49 & 0.08 & 5.3 & 1.40 & 0.14 & 10.0 \\
\hline $\mathrm{Tm}$ & 0.212 & 0.011 & 5.2 & 0.21 & 0.02 & 8.8 \\
\hline $\mathrm{Yb}$ & 1.46 & 0.09 & 6.2 & 1.41 & 0.14 & 9.9 \\
\hline $\mathrm{Lu}$ & 0.22 & 0.03 & 14.3 & 0.20 & 0.02 & 10.3 \\
\hline Hf & 0.34 & 0.08 & 24.0 & 0.34 & 0.04 & 10.5 \\
\hline Ta & 0.019 & 0.009 & 45.7 & 0.019 & 0.002 & 10.5 \\
\hline $\mathrm{Pb}$ & 0.21 & 0.04 & 16.8 & 0.33 & 0.06 & 19.3 \\
\hline Th & 0.012 & 0.005 & 45.1 & 0.008 & 0.002 & 25.0 \\
\hline $\mathrm{U}$ & 0.009 & 0.004 & 42.9 & 0.012 & 0.002 & 12.7 \\
\hline
\end{tabular}

U: Uncertainty at $95 \%$ confidence level; \% var.: percent of variability calculated. 
Table 13. Mean obtained values for 6 determinations in $\mathrm{mg} \cdot \mathrm{kg}^{-1}$ for komatiite glass GOR-132G, compared with values quoted in [15].

\begin{tabular}{|c|c|c|c|c|c|c|}
\hline \multirow{2}{*}{ Elements } & \multicolumn{3}{|c|}{ Obtained values } & \multicolumn{3}{|c|}{ Jochum et al. [15] } \\
\hline & Mean & sd & $\%$ rsd & Mean & $\mathrm{U}$ & $\%$ var \\
\hline $\mathrm{Li}$ & 8.18 & 0.48 & 5.8 & 9.6 & 1.0 & 10.4 \\
\hline Sc & 38.9 & 2.87 & 7.4 & 37.2 & 1.6 & 4.3 \\
\hline $\mathrm{Ti}$ & 2090 & 103 & 4.9 & 1954 & 144 & 7.4 \\
\hline V & 185 & 8 & 4.5 & 219 & 25 & 11.4 \\
\hline $\mathrm{Cr}$ & 2257 & 120 & 5.3 & 2640 & 207 & 7.8 \\
\hline $\mathrm{Mn}$ & 1056 & 50 & 4.7 & 1138 & 108 & 9.5 \\
\hline Co & 84.8 & 4.1 & 4.8 & 94.5 & 10.7 & 11.3 \\
\hline $\mathrm{Ni}$ & 994 & 43 & 4.4 & 1187 & 58 & 4.9 \\
\hline $\mathrm{Cu}$ & 169 & 8 & 4.9 & 208 & 20 & 9.6 \\
\hline $\mathrm{Zn}$ & 54.7 & 2.7 & 4.9 & 79.9 & 16.2 & 20.3 \\
\hline $\mathrm{Ga}$ & 9.28 & 0.59 & 6.4 & 10.1 & 1.3 & 12.9 \\
\hline $\mathrm{Rb}$ & 1.59 & 0.13 & 8.2 & 2.1 & 0.22 & 10.5 \\
\hline $\mathrm{Sr}$ & 14.7 & 0.9 & 6.3 & 15.1 & 1.3 & 8.6 \\
\hline $\mathrm{Y}$ & 12.6 & 0.9 & 6.8 & 13.0 & 1.0 & 7.7 \\
\hline $\mathrm{Zr}$ & 9.44 & 0.77 & 8.2 & 9.7 & 0.8 & 8.2 \\
\hline $\mathrm{Nb}$ & 0.06 & 0.02 & 27.6 & 0.07 & 0.03 & 37.7 \\
\hline Mo & 25.0 & 2.0 & 8.0 & 29.9 & 4.0 & 13.4 \\
\hline Sn & 0.35 & 0.16 & 46.5 & 0.34 & 0.09 & 26.5 \\
\hline $\mathrm{Sb}$ & 0.07 & 0.03 & 44.0 & 0.04 & 0.02 & 50.0 \\
\hline Cs & 6.26 & 0.14 & 2.2 & 7.12 & 1.23 & 17.3 \\
\hline $\mathrm{Ba}$ & 0.73 & 0.10 & 13.2 & 0.82 & 0.14 & 17.6 \\
\hline $\mathrm{La}$ & 0.084 & 0.010 & 12.1 & 0.084 & 0.006 & 7.1 \\
\hline Ce & 0.34 & 0.03 & 8.0 & 0.40 & 0.04 & 10.8 \\
\hline Pr & 0.082 & 0.006 & 7.0 & 0.087 & 0.006 & 6.9 \\
\hline $\mathrm{Nd}$ & 0.70 & 0.07 & 9.3 & 0.68 & 0.03 & 5.0 \\
\hline $\mathrm{Sm}$ & 0.50 & 0.07 & 14.3 & 0.50 & 0.03 & 6.3 \\
\hline $\mathrm{Eu}$ & 0.27 & 0.02 & 8.6 & 0.25 & 0.02 & 6.3 \\
\hline Gd & 1.13 & 0.11 & 10.0 & 1.16 & 0.09 & 7.8 \\
\hline $\mathrm{Tb}$ & 0.27 & 0.03 & 12.8 & 0.26 & 0.02 & 7.6 \\
\hline Dy & 2.08 & 0.14 & 6.9 & 2.16 & 0.14 & 6.5 \\
\hline Ho & 0.49 & 0.07 & 14.2 & 0.49 & 0.04 & 7.1 \\
\hline Er & 1.56 & 0.14 & 9.1 & 1.53 & 0.10 & 6.5 \\
\hline $\mathrm{Tm}$ & 0.24 & 0.03 & 11.4 & 0.23 & 0.02 & 7.3 \\
\hline $\mathrm{Yb}$ & 1.54 & 0.12 & 7.5 & 1.60 & 0.09 & 5.6 \\
\hline $\mathrm{Lu}$ & 0.23 & 0.02 & 10.3 & 0.24 & 0.02 & 8.0 \\
\hline $\mathrm{Hf}$ & 0.28 & 0.07 & 24.3 & 0.35 & 0.04 & 10.0 \\
\hline Ta & 0.035 & 0.002 & 5.8 & 0.030 & 0.003 & 10.0 \\
\hline $\mathrm{Pb}$ & 17.1 & 0.80 & 4.7 & 19.5 & 2.8 & 14.4 \\
\hline Th & 0.007 & 0.005 & 77.4 & 0.009 & 0.003 & 33.3 \\
\hline U & 0.035 & 0.006 & 16.6 & 0.049 & 0.010 & 20.4 \\
\hline
\end{tabular}

U: Uncertainty at 95\% confidence level; \% var.: percent of variability calculated. 


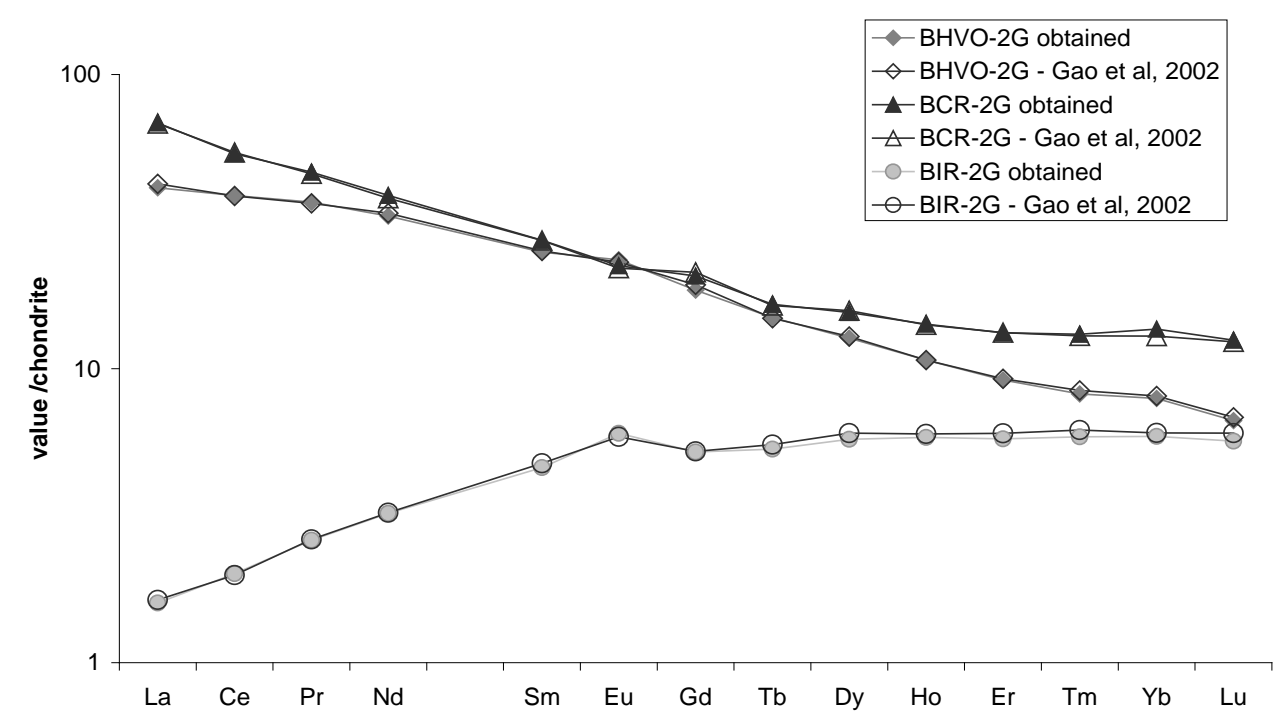

Figure 2. Variation diagrams obtained for the REE contents in the BHVO-2G, BCR-2G and BIR-1G basaltic glasses, normalized with C1 chondrite [16] [17].

Figures 3-5 show the variations in obtained and preferred mean values for the Max Planck RM's (basaltic glasses KL-2G and ML-3BG, andesite StHs6/80G and quartz diorite T-1G, komatiites GOR-128 and GOR$132 \mathrm{G})$. In these diagrams the observed errors are calculated for our obtained results compared with the preferred values for these materials cited in [17].

Variations diagrams for REE are presented in Figures 6-8 normalized with respect to the C1 chondrite [16] [17].

Figure 3 shows that most of the obtained values are placed between $85 \%$ and $115 \%$ of the expected mean values; the exception is Mo in KL-2G. The values obtained for the REE in basalts ML-3BG and KL-2G (Figure 6) are very close to the expected ones.

Some obtained values shown in Figure 4 are higher than the recommended figures cited for the medium to heavy REE and for Y, Zr, Mo and Ti in the quartz diorite T-1G, but lower than the recommended figures for Cr, $\mathrm{Pb}, \mathrm{Ni}$ and $\mathrm{Zn}$. These results may be caused by some tiny inhomogeneity of the reference material, locally concentrating heavy REE and HFSE and diluting the concentration of elements like $\mathrm{Cr}, \mathrm{Pb}, \mathrm{Ni}, \mathrm{Zn}$ and $\mathrm{Sn}$, as shown by [18] for the RM ATHO-1G.

The obtained values compared with the expected ones for the andesite glass StHs6/80G are somewhat outside the limit of $\pm 15 \%$ for the following elements: $\mathrm{Li}, \mathrm{Cr}, \mathrm{Co}, \mathrm{Ni}, \mathrm{Cu}, \mathrm{Ga}, \mathrm{Rb}, \mathrm{Mo}$ and Cs. Chromium and Ni deficiencies are also observed in T-1G, possibly indicating calibration problems, other than inhomogeneous distribution. The REE variation diagram, presented in Figure 7, shows that the obtained values for andesite StHs6/80G is very close to the expected values, while the medium to heavy REE in T-1G shows increased values.

In komatiite GOR-128 (Figure 5), the main elements showing diminished values in relation to the expected ones are $\mathrm{Li}, \mathrm{V}, \mathrm{Cr}, \mathrm{Mn}, \mathrm{Co}, \mathrm{Ni}, \mathrm{Cu}, \mathrm{Zn}, \mathrm{Rb}, \mathrm{Mo}, \mathrm{Cs}, \mathrm{Pb}, \mathrm{U}$. In sample GOR-132G (Figure 5) the same relationship is observed for elements $\mathrm{Ni}, \mathrm{Cu}, \mathrm{Zn}, \mathrm{Rb}, \mathrm{Mo}, \mathrm{Cs}$, Ce, Th and $\mathrm{U}$. These elements show values that are very much increased or diminished when compared with NIST-612, which may not be the best calibration material to be used for this exercise. On the other hand, lack of homogeneity (see [19] and [20]) may be another explanation, possibly caused by quench crystallization of olivine, which could sequester some elements. A larger set of analytical data, not restricted to our limited number of determinations, is needed. The REE variation diagrams presented in Figure 8 (komatiites) show that the obtained values are close to the certified values.

\section{Conclusions}

The obtained values for the several reference materials made available by the USGS and the Max Planck Institute cover a rather wide spectrum of elemental abundances for the over 40 elements determined with the LA-QICPMS technique, the methodology used in this contribution. 


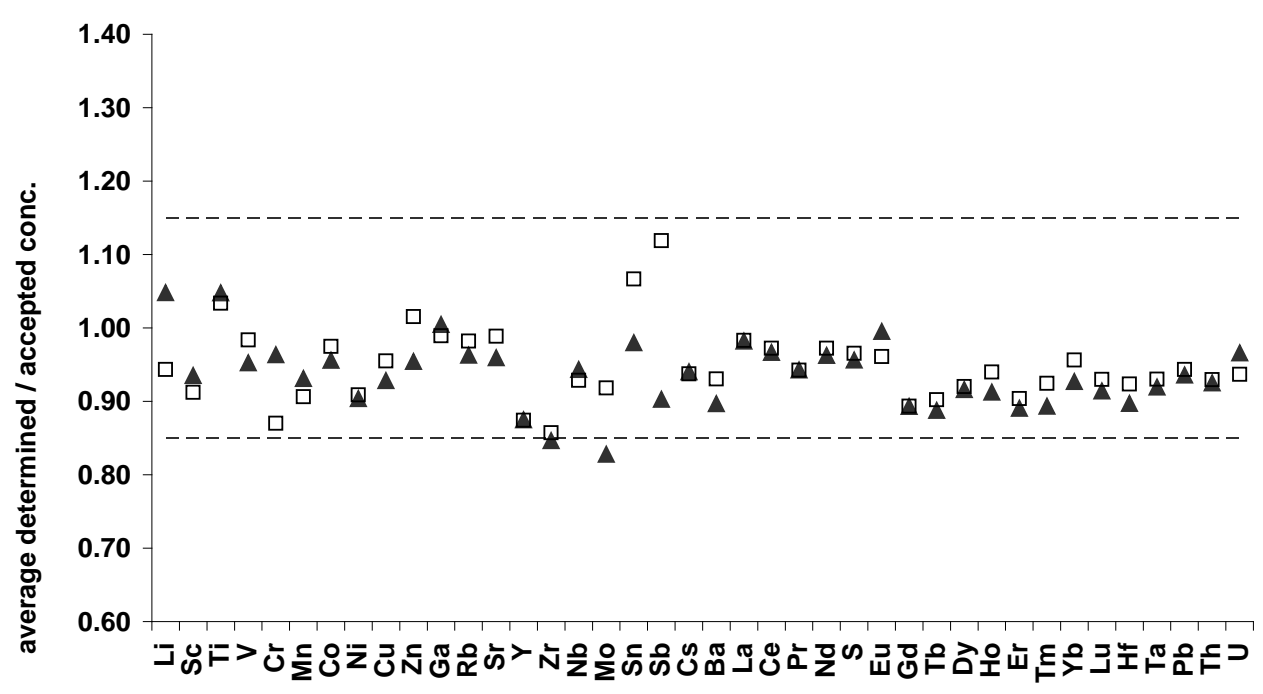

$\Delta \mathrm{KL}-2 \mathrm{G} \quad \square \mathrm{ML}-3 \mathrm{BG}$

Figure 3. Average values obtained in relation to preferred values for several elements in basalt glasses KL-2G and ML-3BG, compared with the mean values cited in [17], cf. Table 8 and Table 9.

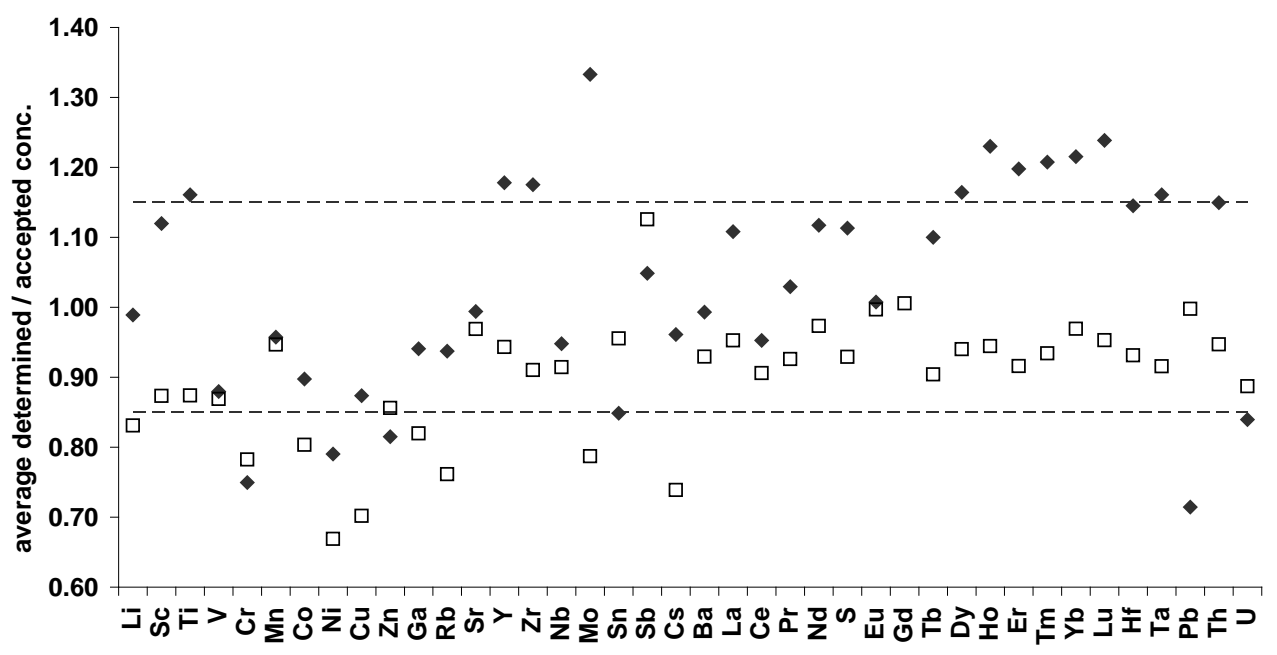

- T-1G 口StHs6-80G

Figure 4. Average values obtained in relation to preferred values for several elements in glasses T-1G (quartz diorite) and StHs6/80G (andesite), compared with the mean certified values cited in [17], cf. Table 10 and Table 11.

The used technique provides high quality results, in terms of analytical accuracy, and is also very fast. The samples can be prepared as thin sections, with a thickness of around 40 to 60 microns, or as mineral grains mounted within polished epoxy resins.

Interferences can be kept at minimum levels, a result achieved with the use of dry plasma, thereby diminishing the influence of agents such as present in aqueous acid systems $\left(\mathrm{O}^{2-}, \mathrm{OH}^{-}, \mathrm{Cl}^{-}, \mathrm{NO}_{3}^{-}\right.$, etc.). However, isotopes such as ${ }^{135} \mathrm{Ba},{ }^{139} \mathrm{La},{ }^{140} \mathrm{Ce}$, and others may interfere on medium and heavy REE, even if the level of oxides is kept below the recommended value of $1 \%$. Increased values of some REE may be caused by the generation of several spurious REE molecular species (e.g. [21]). The point is illustrated in Figure 6, where ${ }^{155}$ Gd may owe its increase to interference by ${ }^{139} \mathrm{La}^{16} \mathrm{O}^{+}$. Careful selection of isotope species is therefore recommended, before relying on one or the other isotopes for analytical calibrations. 


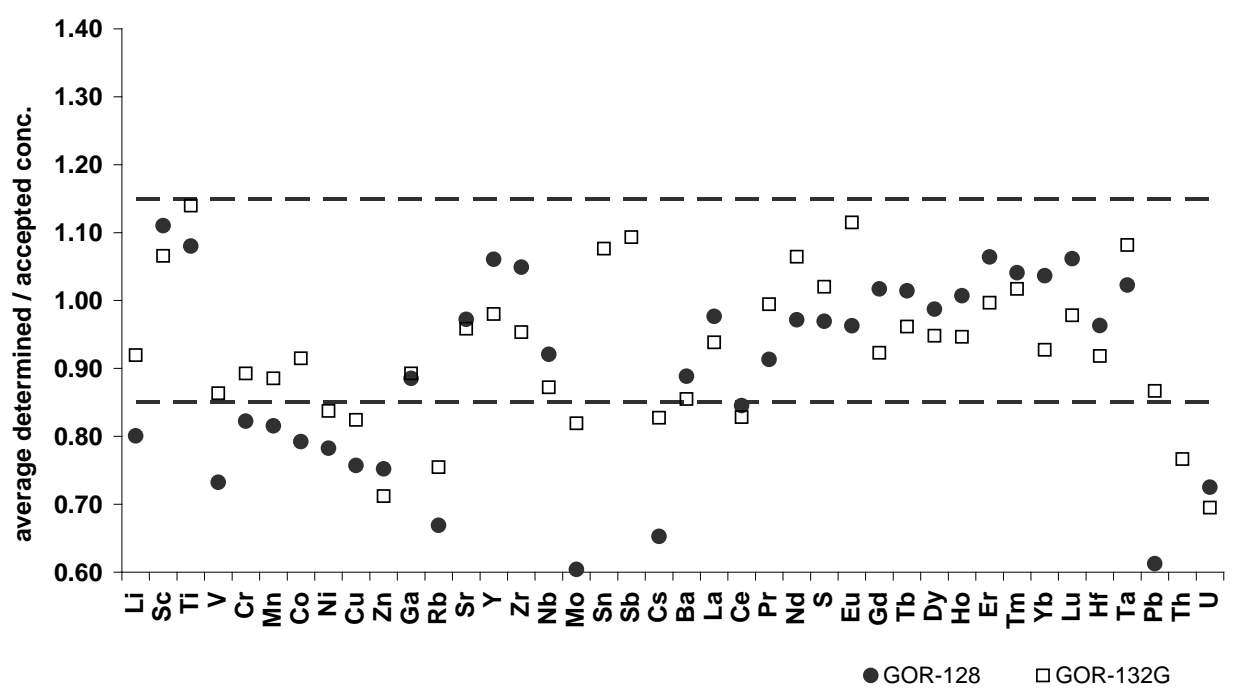

Figure 5. Average values obtained in relation to preferred values for several elements in komatiite glasses GOR-128 and GOR-132G, compared with the mean certified values cited in [17], cf. Table 12 and Table 13.

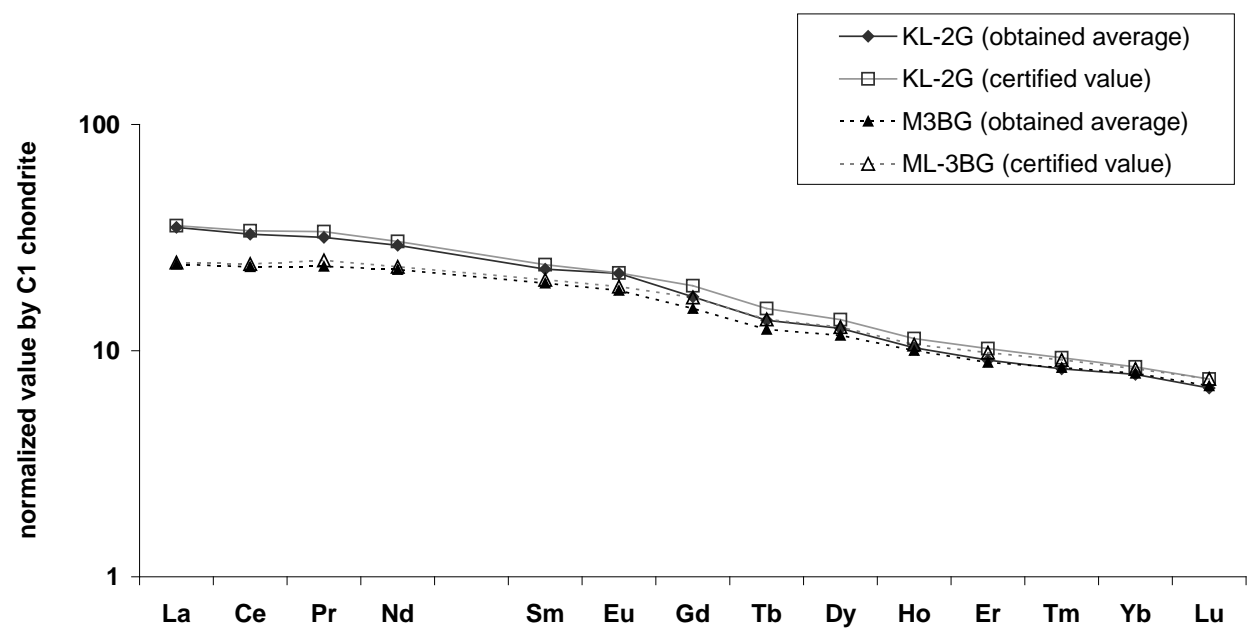

Figure 6. Variation diagrams obtained for the REE contents in basaltic glasses KL-2G and ML-3BG, from the Max Planck Institute, normalized with the C1 chondrite [16] [17].

The rock glasses prepared by the Max Planck Institute cover an adequate spectrum of elements important for studies in igneous petrology, but the materials seem to present a lack of homogeneity for some elements, depending on the analyzed mass and of the spot used for identification [15] and may therefore be the cause for the observed discrepancies in obtained results. More tests are certainly needed to test statistically this hypothesis, in order to certify these glasses as proper equivalents of the USGS materials.

The use of internal standards, especially $\mathrm{MgO}$ and $\mathrm{CaO}$, is a necessary tool to standardize obtained results with our discussed technique. But many minerals may lack sufficient contents of $\mathrm{CaO}$ to be subjected to the LAQ-ICPMS methodology, a fact that limits the scope of the glasses prepared by NIST, with low to very low MgO contents [22] [23]. Another factor to be considered is the possibility of fractionation of many elements, depending on the used internal standard [24]. There is certainly a need to continue the search for an adequate preparation of proper rock standards to be used for LA studies in minerals.

\section{Acknowledgements}

The authors are grateful for the support given by the São Paulo Science Foundation (FAPESP) for the acquisi- 


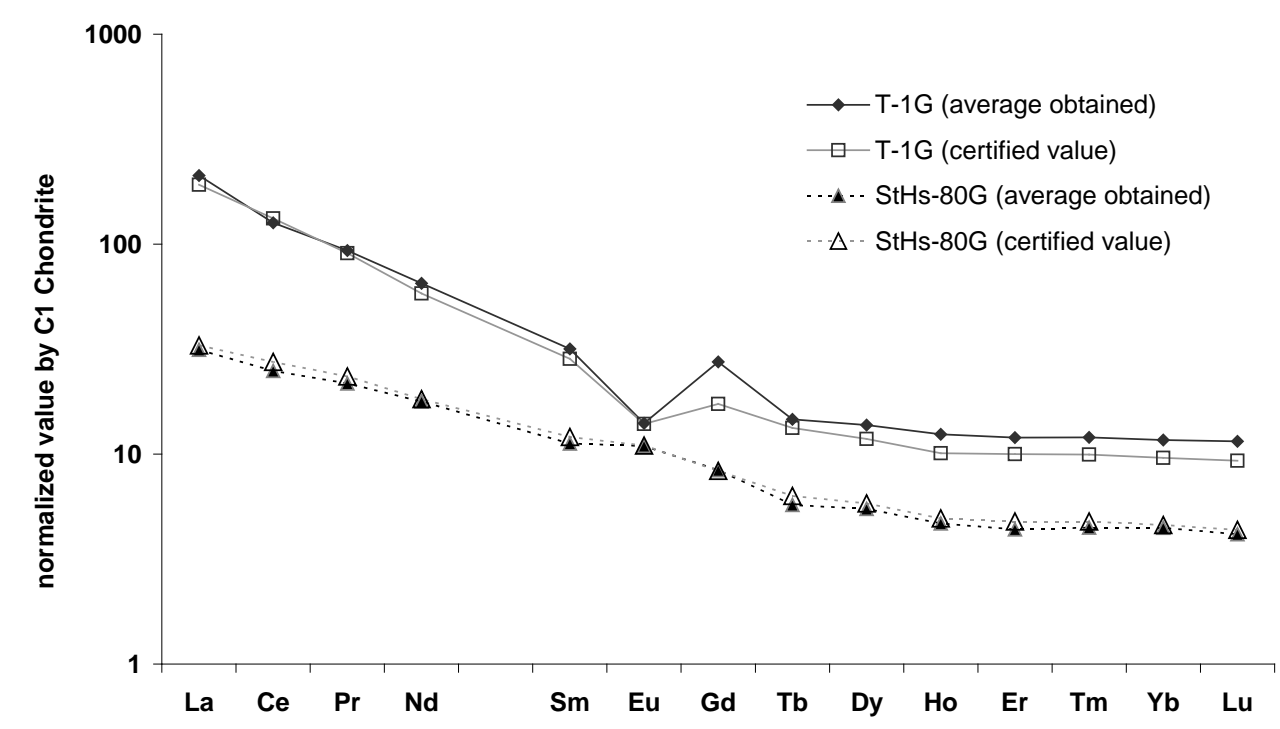

Figure 7. Variation diagrams obtained for the REE contents in basaltic glasses StHs6/80G (andesite) and T-1G (quartz diorite), from the Max Planck Institute, normalized with the C1 chondrite [16] [17].

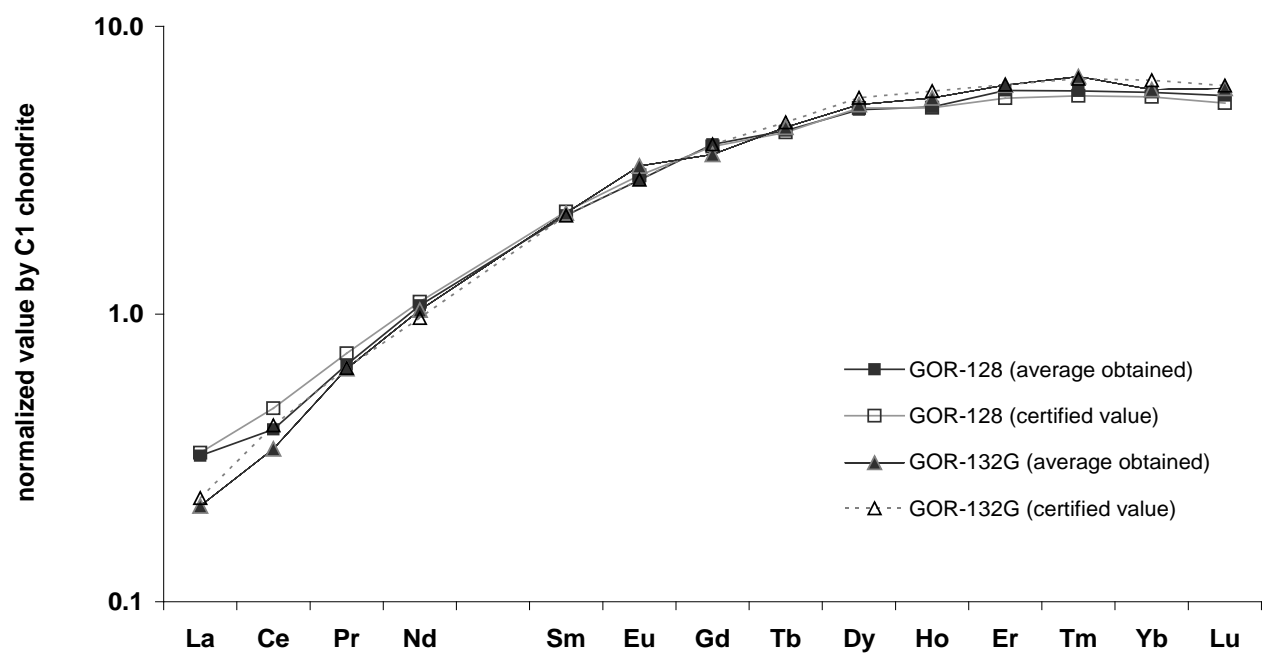

Figure 8. Variation diagrams obtained for the REE contents for komatiite glasses GOR-128 and GOR132G, from the Max Planck Institute, normalized with the C1 chondrite [16] [17].

tion of the spectrometer Q-ICPMS (1999/04824-9) and Laser Ablation System (2004/08856-2) and maintenance and update of the Laboratório de Química e ICP-OES/MS of the Instituto de Geociências, University of São Paulo. This is a contribution of the newly created Geoanalítica-USP Core Facility at the same institute, devoted to mineralogical and petrological studies. Support was also provided by a grant from FAPESP (Nr. 2012/ 06082-6, coordinator, Prof. Excelso Ruberti).

\section{References}

[1] Gunther, D., Bleiner, D., Guillong, M., Hattendorf, B. and Horn, I. (2001) Access to Isotopic and Elemental Composition and Their Distribution in Solid Materials by Laser Ablation-Inductively Coupled Plasma-Mass Spectrometry. Chimia, 55, 778-782.

[2] Montaser, A. (1998) Inductively Coupled Plasma Mass Spectrometry. Wiley-VCH, Washington DC, 964.

[3] Bludy, J. and Wood, B. (1994) Prediction of Crystal-Melt Partition Coefficients from Elastic Module. Nature, 372, 452454. http://dx.doi.org/10.1038/372452a0 
[4] Hanchar, J.M. and Van Westrenen, W. (2007) Rare Earth Element Behaviour in Zircon-Melt Systems. Elements, 3, 37-42. http://dx.doi.org/10.2113/gselements.3.1.37.

[5] Eggins, S.M., Kinsley, L.P.J. and Shelley, J.M.G. (1998) Deposition and Element Fractionation Processes during Atmospheric Pressure Laser Sampling for Analysis by ICP-MS. Applied Surface Science, 127-129, 278-286. http://dx.doi.org/10.1016/S0169-4332(97)00643-0.

[6] Kock, J. and Günther, D. (2011) Reviews of the State-of-the-Art of Laser Ablation Inductively Coupled Plasma Mass Spectrometry. Applied Spectroscopy, 65, 155A-162A. http://dx.doi.org/10.1366/11-06255.

[7] O’Connor, C., Sharp, B.L. and Evans, P. (2006) On-Line Additions of Aqueous Standards for Calibration of Laser Ablation Inductively Coupled Plasma Mass Spectrometry: Theory and Comparison of Wet and Dry Conditions. Journal of Analytical Atomic Spectrometry, 21, 556-565. http://dx.doi.org/10.1039/b600916f.

[8] USGS-United States of Geological Survey (2002) Geochemical Reference Materials and Certificates. http://minerals.cr.usgs.gov/geo_chem_stand/

[9] Jochum, K.P., Weis, U., Stoll, B., Kuzmin, D., Yang, Q., Raczek, I., Jacob, D.E., Stracke, A., Birbaum, K., Frick, D.A., Günther, D. and Enzweiler, J. (2011) Determination of Reference Values for NIST SRM 610-617 Glasses Following ISO Guidelines. Geostandards and Geoanalytical Research, 35, 397-429. http://dx.doi.org/10.1111/j.1751-908X.2011.00120.x.

[10] Jochum, J.P., Willbold, M., Raczek, I., Stoll, B. and Herwing, K. (2005) Chemical Characterization of the USGS Reference Glasses GSA-1G, GSC-1G, GSD-1G, GSE-1G, BCR-2G, BHVO-2G and BIR-1G Using EPMA, ID-TIMS, ID-ICP-MS and LA-ICP-MS. Geostandards and Geoanalytical Research, 29, 285-302. http://dx.doi.org/10.1111/j.1751-908X.2005.tb00901.x

[11] Gao, S., Liu, X., Yuan, H., Hattendorf, B., Gunther, D. and Hu, S.H. (2002) Determination of Forty Two Major and Trace Elements in USGS and NIST SRM Glasses by Laser Ablation-Inductively Coupled Plasma-Mass Spectrometry. Geostandards Newsletter, 26, 181-196. http://dx.doi.org/10.1111/j.1751-908X.2002.tb00886.x

[12] Jackson, S.E. (2001) The Application of Nd:YAG Lasers in LA-ICP-MS. In: Sylvester, P.J., Ed., Laser Ablation-ICP Mass Spectrometry in the Earth Sciences: Principles and Applications, Vol. 29, Short Courses Series, Mineralogical Association of Canada (MAC), 29-45.

[13] Longerich, H.P., Jackson, S.E. and Gunther, D. (1996) Laser Ablation-Inductively Coupled Plasma-Mass Spectrometric Transient Signal Data Acquisition and Analyte Concentration Calculation. Journal of Analytical Atomic Spectrometry, 11, 899-904. http://dx.doi.org/10.1039/ja9961100899.

[14] Klaus Peter Jochum, K.O., Nohl, U., Herwig, K., Lammel, E., Stoll, B. and Hofmann, A.W. (2005) GeoReM: A New Geochemical Database for Reference Materials and Isotopic Standards. Geostandards and Geoanalytical Research, 29, 333-338. http://georem.mpch-mainz.gwdg.de http://dx.doi.org/10.1111/j.1751-908X.2005.tb00904.x

[15] Jochum, J.P., Stoll, B., Herwing, K., Willbold, M., Hofmann, A.W., et al. (2006) MPI-DING Reference Glasses for in Situ Microanalysis: New Reference Values for Element Concentrations and Isotope Ratios. Geochemistry, Geophysics, Geosystems, 7, Q02008. http://dx.doi.org/10.1029/2005GC001060

[16] Sun, S. and McDonough, W.F. (1989) Chemical and Isotopic Systematic of Oceanic Basalts: Implications for Mantle Composition and Processes. In: Saunders, A.D. and Norry, M.J., Eds., Magmatism in the Ocean Basins, Special Publication 42, Geological Society of London, London, 313-345.

[17] Rollinson, H. (1993) Using Geochemical Data: Evaluation, Presentation, Interpretation. Longman Scientific \& Technical, London, 352.

[18] Borisova, A.Y., Freydier, R., Polvé, M., Jochum, K.P. and Candaudap, F. (2010) Multi-Elemental Analysis of ATHO-G Rhyolitic Glass (MPI-DING Reference Material) by Femtosecond and Nanosecond LA-ICP-MS: Evidence for Significant Heterogeneity of B, V, Zn, Mo, Sn, Sb, Cs, W, Pt and Pb at the Millimetre Scale. Geostandards and Geoanalytical Research, 34, 245-255. http://dx.doi.org/10.1111/j.1751-908X.2010.00077.x

[19] Jochum, K.P., Dingwell, D.B., Rocholl, A., Stoll, B., Hofmann, A.W., Becker, S., Besmehn, A., Bessette, D., Dietze, H.J., Dulski, P., Erzinger, J., Hellebrand, E., Hoppe, P., Horn, I., Janssens, K., Jenner, G.A., Klein, M., McDonough, W.F., Maetz, M., Mezger, K., Münker, C., Nikogosian, I.K., Pickhardt, C., Raczek, I., Rhede, D., Seufert, H.M., Simakin, S.G., Sobolev, A.V., Spettel, B., Straub, S., Vincze, L., Wallianos, A., Weckwerth, G., Weyer, S., Wolf, D. and Zimmer, M. (2000) The Preparation and Preliminary Characterization of Eight Geological MPI-DING Reference Glasses for In-Situ Microanalysis. Geostandards Newsletter, 24, 87-133. http://dx.doi.org/10.1111/j.1751-908X.2000.tb00590.x

[20] Kempenaers, L., Janssens, K.K., Jochum, K.P., Vincze, L., Vekemans, B., Somogyi, A., Drakopoulos, M. and Adams, F. (2003) Micro-Heterogeneity Study of Trace Elements in USGS, MPI-DING and NIST Glass Reference Materials by Means of Synchrotron Micro-XRF. Journal of Analytical Atomic Spectrometry, 18, 350-357. http://dx.doi.org/10.1039/b212196d 
[21] Aries, S., Valladon, M., Polvé, M. and Dupré, B. (2000) A Routine Method for Oxide and Hydroxide Interference Corrections in ICP-MS Chemical Analysis of Environmental and Geological Samples. Geostandards Newsletter, 24, 19-31. http://dx.doi.org/10.1111/j.1751-908X.2000.tb00583.x

[22] Mank, A.J.G. and Mason, P.R.D. (1999) A Critical Assessment of Laser Ablation ICP-MS as an Analytical Tool for Depth in Silica-Based Glass Samples. Journal of Analytical Atomic Spectrometry, 14, 1143-1153. http://dx.doi.org/10.1039/a903304a

[23] Dulski, P. (2001) Reference Materials for Geochemical Studies: New Analytical Data by ICP-MS and Critical Discussion of Reference Values. Geostandards Newsletter, 25, 87-125. http://dx.doi.org/10.1111/j.1751-908X.2001.tb00790.x

[24] Hu, M.Y., Fan, X.T., Stoll, B., Kuzmin, D., Liu, Y., Liu, Y., Sun, W., Wang, G., Zhan, X.C. and Jochum, K.P. (2011) Preliminary Characterization of New Reference Materials for Microanalysis: Chinese Geological Standards Glasses CGSG-1, CGSG-2, CGSG-4 and CGSG-5. Geostandards and Geoanalytical Research, 35, 235-251. http://dx.doi.org/10.1111/j.1751-908X.2010.00097.x 
Scientific Research Publishing (SCIRP) is one of the largest Open Access journal publishers. It is currently publishing more than 200 open access, online, peer-reviewed journals covering a wide range of academic disciplines. SCIRP serves the worldwide academic communities and contributes to the progress and application of science with its publication.

Other selected journals from SCIRP are listed as below. Submit your manuscript to us via either submit@scirp.org or Online Submission Portal.
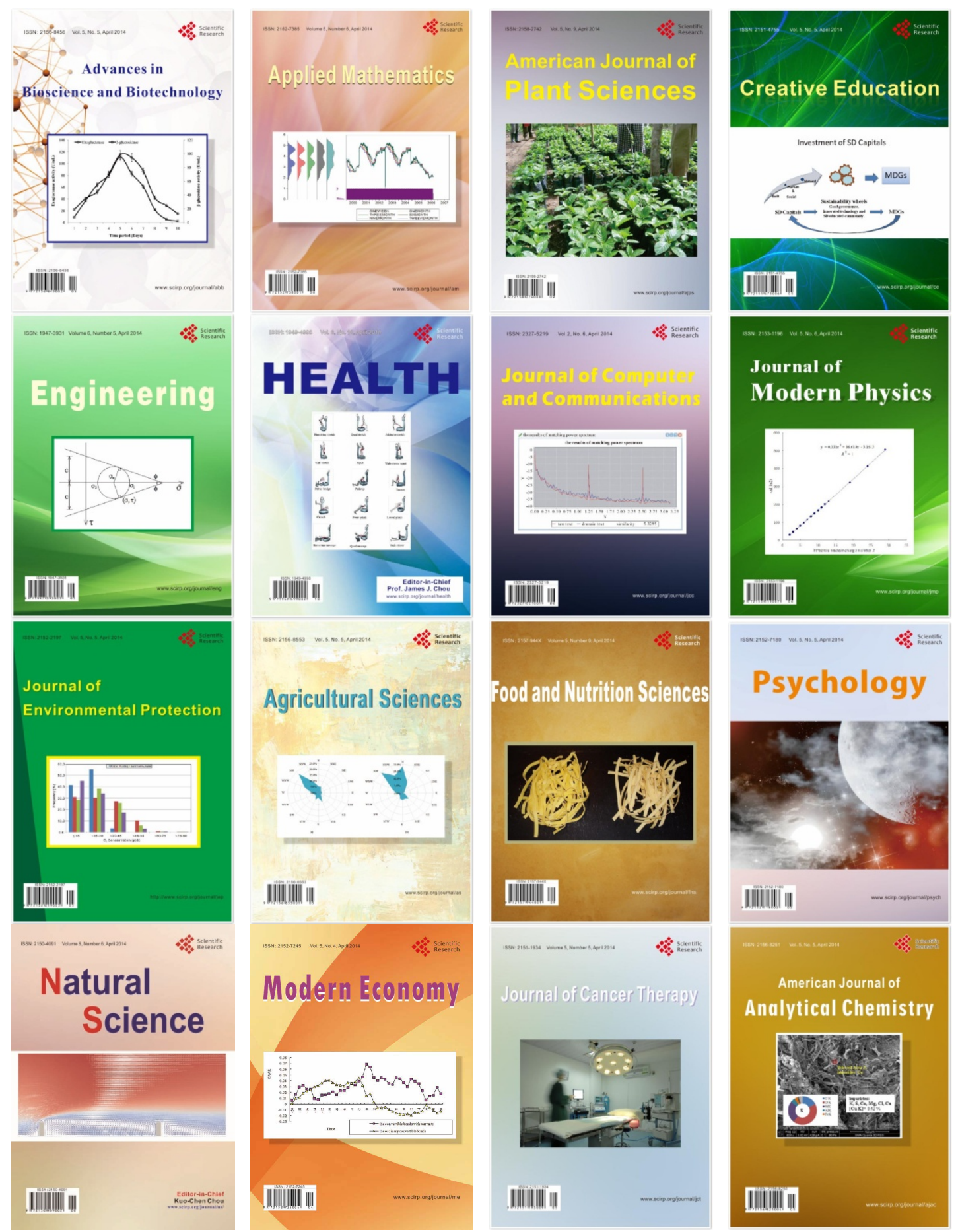